\title{
A Survey of Some Topics Related to Differential Operators
}

\author{
Denise Huet \\ Université de Lorraine, Institut Elie Cartan de Nancy, BP 239, 54506 Vandoeuvre-les-Nancy Cedex, France \\ Correspondence should be addressed to Denise Huet; denise.huet@univ-lorraine.fr \\ Received 26 March 2013; Revised 2 October 2013; Accepted 4 October 2013 \\ Academic Editor: Qingkai Kong \\ Copyright (C) 2013 Denise Huet. This is an open access article distributed under the Creative Commons Attribution License, which \\ permits unrestricted use, distribution, and reproduction in any medium, provided the original work is properly cited.

\begin{abstract}
This paper is the result of investigations suggested by recent publications and completes the work of Huet, 2010. The topics, which are dealt with, concern some spaces of functions and properties of solutions of linear and nonlinear, stationary and evolution differential equations, namely, existence, spectral properties, resonances, singular perturbations, boundary layers, and inertial manifolds. They are presented in the alphabetical order. The aim of this document and of Huet, 2010, is to be a useful reference for (young) researchers in mathematics and applied sciences.
\end{abstract}

\section{Introduction}

The article is divided into several sections entitled: BirmanSchwinger operators; BMO spaces; Bounded variation (functions of); Discrete energy; Dissipative operators; Dynamical systems; Equal- area condition; Inertial manifolds; MathieuHill type equations; Memory (equations with); Nodes, Nodal; Resonances. The development of each entry includes indications on history, definitions, an overview of main results, examples, and applications but is, of course, nonexhaustive. Complements will be found in the references. A prepublication of some entries is presented in Huet [1].

\section{Birman-Schwinger Operator}

Definition 1. Consider the Schrödinger operator

$$
H_{\gamma}=H_{o}+\gamma V(x), \quad H_{o}=(-\Delta)^{l},
$$

acting on $L^{2}\left(\mathbb{R}^{d}\right), 2 l \geq d$, where $x \rightarrow V(x)$ is a real-valued continuous function defined on $\mathbb{R}^{d}$ which is nonnegative and tends to zero, sufficiently fast, as $|x| \rightarrow \infty$ and $\gamma$ is a small negative coupling constant. The operator $H_{o}$ is self-adjoint and its spectrum is $\sigma_{o}=[0,+\infty)$. The Birman-Schwinger operator associated with (1) is the operator

$$
X_{V}(\lambda)=V^{1 / 2} R_{\lambda}\left(H_{o}\right) V^{1 / 2},
$$

where $R_{\lambda}\left(H_{o}\right)$ is the resolvent of $H_{o}$ in $(-\infty, 0)$. For each $\lambda \in(-\infty, 0), X_{V}(\lambda)$ is self-adjoint and compact (cf. Arazy and Zelenko [2]).

Application. In [2], the authors consider the decomposition $X_{V}(\lambda)=\Phi(\lambda)+T(\lambda)$, where $\Phi(\lambda)$ is a finite rank operator and $T(\lambda)$ an Hilbert-Schmidt operator whose norm is uniformly bounded with respect to $\lambda \in(-\delta, 0)$ for some $\delta>0$. An asymptotic expansion of the bottom virtual eigenvalue $\lambda_{o}(\gamma)$ of $H_{\gamma}$, as $\gamma<0$ tends to zero, is deduced from this decomposition: if $d$ is odd, it is of power type, while, when $d$ is even, it involves the log function. Asymptotic estimates are obtained, as $\gamma \uparrow 0$, for the nonbottom virtual eigenvalues of $H_{\gamma},\left\{\lambda_{\mathbf{k}}(\gamma), \mathbf{k} \in \mathbb{Z}_{+}^{d} ; 0<|\mathbf{k}| \leq m\right\}$, where $m=l-((d+1) / 2)$ if $d$ is odd and $m=l-(d / 2)$ if $d$ is even. If $d$ is odd, $\Phi\left(-t^{2 l}\right)$ is a meromorphic operator function, and the leading terms of the asymptotic estimates of $\lambda_{\mathbf{k}}(\gamma)$ are of power type. An algorithm, based on the Puiseux-Newton diagram (cf. Baumgärtel [3]), is proposed for an evaluation of the leading coefficients of these estimates. If $d$ is even, two-sided estimates are obtained for eigenvalues with an exponential rate of decay; the rest of the eigenvalues have a power rate of decay. Estimates of Lieb-Thirring type are obtained for groups of eigenvalues which have the same rate of decay, when $d$ is odd or even. 


\section{BMO Spaces}

\subsection{BMO and Related Spaces}

Definition 2. Let $\sup _{I}$ be the supremum over all cubes $I \subset \mathbb{R}^{n}$ with edges parallel to the coordinate axes, $\ell(I)$ the sidelength of $I$, and $f_{I}$ the mean value of $f$ over $I$. The John-Nirenberg's $\mathrm{BMO}=\mathrm{BMO}\left(\mathbb{R}^{n}\right)$ space (cf. John and Nirenberg [4]) is the space of locally integrable complex-valued functions $f$ defined on $\mathbb{R}^{n}$, such that

$$
\|f\|_{\mathrm{BMO}}=\left(\sup _{I}(\ell(I))^{-n} \int_{I}\left|f(x)-f_{I}\right|^{2} d x\right)^{1 / 2}<\infty .
$$

The space of functions of bounded mean oscillation, modulo constants, equipped with the above norm, is a Banach space.

Definition 3 (real Hardy space $H^{1}\left(\mathbb{R}^{n}\right)$ ). A function $f \in$ $H^{1}\left(\mathbb{R}^{n}\right)$ if and only if

$$
f=\sum c_{j} \alpha_{j}
$$

where $c_{j} \in \mathbb{R}, \sum\left|c_{j}\right|<\infty$, each function $\alpha_{j}$ is supported on a ball $B_{j}$ and has integral zero, and $\sup _{x \in B_{j}}\left|\alpha_{j}(x)\right| \leq 1 /\left|B_{j}\right|$. Functions $\alpha$ that satisfy the above properties are called 1-atom (cf. Stein, [5]). With the norm

$$
\|f\|_{H^{1}}=\inf \sum\left|c_{j}\right|
$$

where the inf is taken on all decompositions of $f$ of the form (4), $H^{1}\left(\mathbb{R}^{n}\right)$ is a Banach space. In Fefferman and Stein [6, Theorem 2], it is proved that the dual of $H^{1}\left(\mathbb{R}^{n}\right)$ is BMO. For definitions and properties of Hardy-spaces $H^{p}\left(\mathbb{R}^{n}\right)$, see [6].

Related spaces are presented in [7].

3.2. BMO Nonlinearity (cf. Byun and Wang [8]). Let $a(\xi, x)$ be a nonlinear, real valued, function on $\mathbb{R}^{n} \times \mathbb{R}^{n}$. For $y \in \mathbb{R}^{n}$ and $\rho>0, B_{\rho}(y)$ denotes the open ball of radius $\rho$ centered at $y$. Set

$$
\bar{a}_{B_{\rho}(y)}(\xi)=\frac{1}{\left|B_{\rho}(y)\right|} \int_{B_{\rho}(y)} a(\xi, x) d x
$$

and define the function $\beta\left(a, B_{\rho}(y)\right)$ by

$$
\beta\left[a, B_{\rho}(y)\right](x)=\sup _{\xi \in \mathbb{R}^{n}} \frac{\left|a(\xi, x)-\bar{a}_{B_{\rho}(y)}(\xi)\right|}{|\xi|+1} .
$$

Definition $4((\delta, R)$-BMO condition). The function $a=$ $a(\xi, x)$ satisfies the $(\delta, R)-B M O$ condition if

$$
\sup _{0<\rho \leq R} \sup _{y \in \mathbb{R}^{n}} \frac{1}{\left|B_{\rho}(y)\right|} \int_{B_{\rho}(y)}\left|\beta\left[a, B_{\rho}(y)\right]\right|^{2}(x) d x \leq \delta^{2} .
$$

Definition 5 (Reifenberg domain). A bounded open set $\Omega$ in $\mathbb{R}^{n}$ is $(\delta, R)$-Reifenberg flat if, for every $x_{o} \in \partial \Omega$ and every $r \in$ $(0, R]$, there exists a coordinate system $\left\{y_{1}, \ldots, y_{n}\right\}$ (which can depend on $r$ and $x_{o}$ ) so that $x_{o}$ is the origin in this coordinate system and that

$$
B_{r}(0) \cap\left\{y_{n}>\delta r\right\} \subset B_{r}(0) \cap \Omega \subset B_{r}(0) \cap\left\{y_{n}>-\delta r\right\}
$$

(cf. [9]).
Applications. (i) Let $\Omega$ be a bounded, open subset of $\mathbb{R}^{n}$, $f \in L^{p}\left(\Omega ; \mathbb{R}^{n}\right), 2 \leq p<\infty, a=a(\xi, x): \mathbb{R}^{n} \times \mathbb{R}^{n} \rightarrow$ $\mathbb{R}^{n}$ is a vector field measurable in $x$ for almost every $\xi$ and continuous in $\xi$ for each $x$. In [8], the authors consider the nonlinear boundary value problem

$$
\operatorname{div} a(\nabla u, x)=\operatorname{div} f, \quad \text { in } \Omega \quad u=0 \quad \text { on } \partial \Omega .
$$

The following conditions are imposed on $a(\xi, x)$ :

$$
[a(\xi, x)-a(\eta, x)] \cdot(\xi-\eta) \geq c_{o}|\xi-\eta|^{2}
$$

for all $\xi, \eta \in \mathbb{R}^{n}$ and almost every $x \in \Omega$,

$$
|a(\xi, x)| \leq c_{1}(1+|\xi|)
$$

for all $\xi \in \mathbb{R}^{n}$ and almost $x \in \mathbb{R}^{n}$, and

$$
\left|\nabla_{\xi} a(\xi, x)\right| \leq c_{2} \quad \text { for any } \xi \in \mathbb{R}^{n} \text { and almost every } x \in \mathbb{R}^{n}
$$

for some positive constants $c_{o}, c_{1}$, and $c_{2}$. Then it is proved that there exists $\delta>0$ such that, if a satisfies the $(\delta, R)$-BMO condition and $\Omega$ is $(\delta, R)$-Reifenberg flat, the weak solution $u \in H_{o}^{1}(\Omega)$ to $(10)$ belongs to $W_{o}^{1, p}(\Omega)$ with the estimate

$$
\|\nabla u\|_{L^{p}(\Omega)} \leq C\left(\|f\|_{L^{p}(\Omega)}+1\right),
$$

where $C$ is independent of $u$ and $f$.

(ii) In [9], S-S Byun extends the previous results to Orlicz spaces. He recalls the following definitions.

Definition 6. A positive function $\phi$ defined on $[0, \infty)$ is called a Young function if it is increasing, convex and satisfies

$$
\begin{gathered}
\phi(0)=0, \quad \phi(\infty)=\lim _{t \rightarrow \infty} \phi(t)=\infty, \\
\lim _{t \rightarrow 0} \frac{\phi(t)}{t}=0, \quad \lim _{t \rightarrow \infty} \frac{\phi(t)}{t}=\infty .
\end{gathered}
$$

Definition 7. One says that the Young function $\phi \in \Delta_{2} \cup \nabla_{2}$ if it satisfies the following conditions:

$$
\begin{gathered}
\phi(2 t) \leq \kappa \phi(t), \quad \forall t \geq 0, \\
2 t_{o} \phi(t) \leq \phi\left(t_{o} t\right), \quad \forall t>0,
\end{gathered}
$$

for some numbers $\kappa, t_{o}>1$.

Definition 8. Let $\phi \in \Delta_{2} \cup \nabla_{2}$ be a Young function. The Orlicz space $L^{\phi}(\Omega)$ is the linear space of all measurable functions $v: \Omega \rightarrow \mathbb{R}$ satisfying

$$
\int_{\Omega} \phi(|v|) d x<\infty
$$

Equipped with the norm

$$
\|v\|_{L^{\phi}(\Omega)}=\inf \left\{\lambda>0: \int_{\Omega} \phi\left(\frac{|v|}{\lambda}\right) d x \leq 1\right\},
$$

$L^{\phi}(\Omega)$ is a Banach space. 
The following result is proved in [9]: let $\phi \in \Delta_{2} \cup \nabla_{2}$ be a Young function. There exist a small $\delta>0$ and a positive constant $C$ such that, if the nonlinearity $a$ satisfies (11), (12), (13), and (8), if $\Omega$ satisfies (9), and if $|f|^{2} \in L^{\phi}(\Omega)$, then the unique weak solution $u \in H_{o}^{1}(\Omega)$ to (10) satisfies $|\nabla u|^{2} \in$ $L^{\phi}(\Omega)$, with the estimate

$$
\int_{\Omega} \phi\left(|\nabla u|^{2}\right) d x \leq C\left(1+\int_{\Omega} \phi\left(|f|^{2}\right) d x\right) .
$$

Remark 9. If $p>2$ and $\phi(t)=t^{p / 2}$ inequality (19) reduces to only (14).

\section{4. (Functions of) Bounded Variation}

The notion of functions of bounded variation is closely related to the notion of measure and the following usual definitions are useful.

\subsection{Measures}

Definition 10. Let $\Omega$ be an open subset of $\mathbb{R}^{n}$, and $\mathscr{C}_{c}(\Omega, \mathbb{C})$ $\left[\right.$ resp., $\left.\mathscr{C}_{c}(\Omega, \mathbb{R})\right]$ the space of continuous functions $\varphi: \Omega \rightarrow$ $\mathbb{C}[$ resp., $\mathbb{R}]$ with compact support in $\Omega$.

(i) A measure $\mu$ on $\Omega$ is a linear functional

$$
\mu: \varphi \in \mathscr{C}_{c}(\Omega, \mathbb{C}) \longrightarrow \mu(\varphi)(\operatorname{or}\langle\mu, \varphi\rangle) \in \mathbb{C}
$$

that is continuous; in the following sense, for all $K$ compact in $\Omega$, there exists a constant $C_{K}$ such that

$$
|\langle\mu, \varphi\rangle| \leq C_{K}\|\varphi\|_{\infty}
$$

for all $\varphi \in \mathscr{C}_{c}(\Omega, \mathbb{C})$ whose support is contained in $K$. We write $\mu \in \mathscr{M}(\Omega)$. In [10], Schwartz introduces a topology on $\mathscr{C}_{c}(\Omega, \mathbb{C})$ and $\mu$ is continuous on this topological space.

(ii) $\mu$ is said to be bounded on $\Omega$ if, in (21), the constant is independent of $K$. The space of bounded measures on $\Omega$ is denoted by $\mathscr{M}^{1}(\Omega)$.

(iii) The conjugate $\bar{\mu}$ of $\mu$ is given by

$$
\langle\bar{\mu}, \varphi\rangle=\overline{\langle\mu, \bar{\varphi}\rangle} .
$$

(iv) $\mu$ is called real if $\langle\mu, \varphi\rangle \in \mathbb{R}$, for all $\varphi \in \mathscr{C}_{c}(\Omega, \mathbb{R})$.

(v) $\mu$ is called positive if $\langle\mu, \varphi\rangle \geq 0$, for all $\varphi \in \mathscr{C}_{c}(\Omega$, $\mathbb{R}), \varphi \geq 0$.

Definition 11 (absolute value of a measure). If $\mu$ is a complex or real measure, its absolute value, denoted by $|\mu|$, is the map

$$
\begin{gathered}
\psi \in \mathscr{C}_{c}(\Omega, \mathbb{R}), \\
\psi \geq 0 \longrightarrow\langle|\mu|, \psi\rangle=\sup _{\varphi \in \mathscr{C}_{c}(\Omega, \mathbb{C}),|\varphi| \leq \psi}\{|\langle\mu, \varphi\rangle|\} .
\end{gathered}
$$

Definition 12 (total variation of a positive bounded measure). Let $\mu$ be a positive, bounded measure on $\Omega$. Its total variation, denoted by $|\mu|_{\Omega}$ or $\int_{\Omega} \mu$, is

$$
|\mu|=\sup _{\varphi \in \mathscr{C}_{c}(\Omega, \mathbb{R}), 0 \leq \varphi \leq 1} \mu(\varphi) .
$$

4.2. Space $\mathrm{BV}(I, \mathbb{R})$ or $\mathrm{BV}(I)$. Let $I=(a, b)$ be an interval in $\mathbb{R}$. The following alternative definitions are well known. A function $f: x \in I \rightarrow \mathbb{R}$ is of bounded variation:

(i) if it can be expressed in the form $\phi-\psi$, where $\phi, \psi$ are nondecreasing bounded functions,

(ii) if the interval $(a, b)$ is divided up by points

$$
a=x_{o}<x_{1}<\cdots<x_{n-1}<x_{n}=b,
$$

then, there exists a constant $C>0$, independent of the mode of division, such that

$$
\sum_{\nu=0}^{n-1}\left|f\left(x_{\nu+1}\right)-f\left(x_{\nu}\right)\right|<C
$$

and the upper bound of the sum (26) is called the total variation of $f$ on $I$ and is denoted by $V(f, I)$.

For these definitions, see Titchmarsh [11, page 355] and Riesz and Sz-Nagy [12, page 10].

We have also the following proposition: let $f \in L_{\text {loc }}^{1}(\mathbb{R})$. Then $f$ defines a distribution $T=T_{f} \in \mathscr{D}^{\prime}(\mathbb{R})$. In Schwartz $[10$, page 53$]$ it is proved that, in order for the derivative of $T$ to be a measure, it is necessary and sufficient that $f$ is of bounded variation on every finite interval.

4.3. Space $\operatorname{BV}(\Omega, \mathbb{R})$ or $\operatorname{BV}(\Omega)$. Let $\Omega$ be an open set in $\mathbb{R}^{n}$. The following definitions are equivalent (cf. Brezis [13, page 153]).

A function $f \in W_{1}^{1}(\Omega)$ is of bounded variation

(i) if all first derivatives of $f$, in the distributional sense, that is, in $\mathscr{D}^{\prime}(\Omega)$, are bounded measures,

(ii) if there exists a constant $C$ such that

$$
\left|\int_{\Omega} f \frac{\partial \phi}{\partial x_{i}} d x\right| \leq C\|\phi\| L^{\infty}(\Omega) \quad \forall \phi \in C_{c}^{\infty}(\Omega), i=1, \ldots, n,
$$

(iii) if there exists a constant $C$ such that

$$
\left\|\tau_{h} f-f\right\|_{L^{1}(\omega)} \leq C|h|
$$

for all open set $\omega \subset \Omega$ and all $h \in \mathbb{R}^{n}$ with $|h|<$ $\operatorname{dist}(\omega, C \Omega)$. Moreover, in (27) and (28) we can take $C=\|\nabla f\|_{L^{1}(\Omega)}$. Here $\tau_{h} f(x)=f(x+h)$, and $\nabla f$ is the distributional gradient of $f$.

We have also the following proposition: a function $f \in$ $L^{1}(\Omega)$ is of bounded variation if its first distributional derivatives are bounded measures. Then, the gradient $\nabla f$ is a bounded, vector-valued measure whose absolute value $|\nabla f|$ is the map

$$
\begin{aligned}
& \psi \in \mathscr{C}_{c}(\Omega, \mathbb{R}), \\
& \psi \geq 0 \longrightarrow\langle|\nabla f|, \psi\rangle=\sup _{\varphi \in \mathscr{C}_{c}\left(\Omega, \mathbb{C}^{n}\right), \Sigma_{i}^{n}\left|\varphi_{i}\right|^{2} \leq \psi^{2}}\{|\langle\nabla f, \varphi\rangle|\},
\end{aligned}
$$


where $\langle\nabla f, \varphi\rangle=\sum_{i}^{n}\left(\partial f / \partial x_{i}\right) \varphi_{i}$ and $|\nabla f|$ is a bounded positive measure. The total variation of $|\nabla f|$ is

$$
\int_{\Omega}|\nabla f|=\|\nabla f\|_{\Omega}=\sup _{\psi \in \mathscr{C}_{c}(\Omega, \mathbb{R}), 0 \leq \psi \leq 1}\langle|\nabla f|, \psi\rangle
$$

(cf. F. Demengel and G. Demengel [14, page 303]).

4.4. Space $\operatorname{BV}\left(\Omega, \mathbb{R}^{n}\right)$. This section is already presented in [7]. Let $\Omega$ be an open subset of $\mathbb{R}^{d}$ with a smooth boundary. A function $f \in L_{\text {loc }}^{1}\left(\Omega, \mathbb{R}^{n}\right)$ has a bounded variation, that is, $f \in \operatorname{BV}\left(\Omega, \mathbb{R}^{n}\right)$, if $\nabla f$, in the distributional sense, is a vectorvalued Radon measure of finite total mass. Let $|\mathbf{f}|_{\mathbf{B V}}=\int_{\Omega}|\nabla \mathbf{f}|$ be a BV-seminorm. In Dávila [15], the following property of $|f|_{\text {BV }}$ is proved: there exists a positive constant $K$, which depends on $d$, such that, for every family of nonnegative radial mollifiers $\rho_{\epsilon} \in L_{\text {loc }}^{1}\left((0, \infty), \mathbb{R}_{+}\right)$satisfying

$$
\int_{0}^{\infty} \rho_{\epsilon}(r) r^{d-1} d r=1, \quad \lim _{\epsilon \rightarrow 0} \int_{\delta}^{\infty} \rho_{\epsilon}(r) r^{d-1} d r=0
$$

$$
\forall \delta>0,
$$

we have

$$
\begin{array}{r}
\lim _{\epsilon \rightarrow 0} \int_{\Omega^{2}} \frac{|f(x)-f(y)|}{|x-y|} \rho_{\epsilon}(|x-y|) d x d y=K|f|_{\mathrm{BV}}, \\
\forall f \in \operatorname{BV}\left(\Omega, \mathbb{R}^{n}\right) .
\end{array}
$$

4.5. Application. In [16], B. Merlet shows, by means of the above property of $|f|_{\mathrm{BV}}$, that, if $u \in \operatorname{BV}\left(\Omega, S^{1}\right)$, there exists a lifting $\varphi \in \operatorname{BV}(\Omega, \mathbb{R})$ of $u$ (i.e., $u(x)=e^{i \varphi(x)}$, for all $x \in \Omega$ ) such that $|\varphi|_{\mathrm{BV}} \leq 2|u|_{\mathrm{BV}}$.

\section{Discrete Energy}

Let $\omega_{N}=\left(x_{1}, \ldots, x_{N}\right), N \geq 2$ be a set of $N$ points on the unit-sphere $S^{2}=\left\{x \in \mathbb{R}^{3},|x|=1\right\}$.

5.1. Discrete Energy of $\omega_{N}$. Different discrete energies are associated with $\omega_{N}$. Let $\alpha \in \mathbb{R},-2<\alpha<2$.

Definition 13. The Coulomb [resp., logarithmic] energy associated with $\omega_{N}$ is

$$
\mathscr{H}_{C}\left(\omega_{N}\right)=\sum_{i=1}^{N} \sum_{j=i+1}^{N} \frac{1}{\left|x_{i}-x_{j}\right|}
$$

[resp., $\left.\mathscr{H}_{L}\left(\omega_{N}\right)=-\sum_{i=1}^{N} \sum_{j=i+1}^{N} \log \left|x_{i}-x_{j}\right|\right]$.

Definition 14. More generally, the $\alpha$-energy associated with $\omega_{N}$ is

$$
E\left(\alpha, \omega_{N}\right)= \begin{cases}\sum_{i=1}^{N} \sum_{j=i+1}^{N}\left|x_{i}-x_{j}\right|^{\alpha} & \text { if } \alpha \neq 0, \\ \mathscr{H}_{L}\left(\omega_{N}\right) & \text { if } \alpha=0 .\end{cases}
$$

Remark 15. We have

$$
\mathscr{H}_{C}\left(\omega_{N}\right)=E\left(-1, \omega_{N}\right) .
$$

Remark 16. $E\left(\alpha, \omega_{N}\right)$ is the energy of the $N$ points $x_{1}, \ldots, x_{N}$, on the surface of the sphere, interacting through a potential $V=r^{\alpha}$ (cf. Rakhmanov et al. [17]).

The article [17] is devoted to extremal energy for $\omega_{N}$ :

$$
\mathscr{E}(\alpha, N)= \begin{cases}\inf _{\omega_{N} \in S^{2}} E\left(\alpha, \omega_{N}\right) & \text { if } \alpha \leq 0, \\ \sup _{\omega_{N} \in S^{2}} E\left(\alpha, \omega_{N}\right) & \text { if } \alpha>0 .\end{cases}
$$

Bounds for $\mathscr{E}(\alpha, N),-2<\alpha<2$ and explicit formula for $N$ points on $S^{2}$ that yields good estimates for $\mathscr{E}(\alpha, N)$ are obtained. The authors point out important applications of the determination of $\mathscr{E}(\alpha, N)$ to geometry, chemistry, physics, and crystallography and give references for the history of related researches.

5.2. A More General Discrete Energy-Like Function on the Unit Sphere. In [18], Cheviakov et al. introduce the following definition.

Definition 17. The discrete energy-like function associated with $\omega_{N}$ is

$$
\begin{aligned}
\mathscr{H}\left(\omega_{N}\right)= & \mathscr{H}_{C}\left(\omega_{N}\right)+\frac{1}{2} \mathscr{H}_{L}\left(\omega_{N}\right) \\
& -\frac{1}{2} \sum_{i=1}^{N} \sum_{j=i+1}^{N} \log \left(2+\left|x_{i}-x_{j}\right|\right) .
\end{aligned}
$$

Application. In [18], $\mathscr{H}\left(\omega_{N}\right)$ is related to the mean first passage time (MFPT) $v(x)$ for a Brownian particle in the unit ball $\Omega$ in $\mathbb{R}^{3}$ that contains $N$ small locally circular absorbing windows $\partial \Omega_{\epsilon_{j}}$ on its boundary $\partial \Omega=S^{2}$. Set $\partial \Omega_{a}=\bigcup_{j=1}^{N} \partial \Omega_{\epsilon_{j}}$. The function $v(x)$ is solution to the Dirichlet-Neumann problem:

$$
\begin{gathered}
\Delta v=-\frac{1}{D} \quad \text { on } \Omega, \\
v=0 \quad \text { on } \partial \Omega_{a}, \quad \frac{\partial v}{\partial n}=0 \quad \text { on } \frac{\partial \Omega}{\partial \Omega_{a}},
\end{gathered}
$$

where $D$ is a diffusivity coefficient. The authors obtain threeterm asymptotic expansions for $v(x)$, for the average MFPT $\bar{v}=(1 /|\Omega|) \int_{\Omega} v(x) d x$, and for the principal eigenvalue $\lambda$ of the Laplacian associated with the boundary conditions (38), when the area $\left|\partial \Omega_{\epsilon_{j}}\right| \sim \pi \epsilon^{2} a_{j}^{2}, \partial \Omega_{\epsilon_{j}} \rightarrow x_{j} \in S^{2}$ as $\epsilon \rightarrow 0$, 
$j=1, \ldots, N$ and $\left|x_{i}-x_{j}\right|=\mathcal{O}(1), i \neq j$. For instance, when the windows have common radius $\epsilon \ll 1$, they obtain

$$
\begin{aligned}
& \bar{v}=\frac{|\Omega|}{4 \epsilon D N}[1+\frac{\epsilon}{\pi} \log \left(\frac{2}{\epsilon}\right) \\
&+ \frac{\epsilon}{\pi}\left(-\frac{9 N}{5}+2(N-2) \log 2+\frac{3}{2}\right) \\
&+\left.\frac{4}{N} \mathscr{H}\left(x_{1}, \ldots, x_{N}\right)+\mathcal{O}\left(\epsilon^{2} \log \epsilon\right)\right], \\
& \lambda \sim \frac{1}{D \bar{v}} .
\end{aligned}
$$

Moreover, $\bar{v}$ is minimized and the corresponding $\lambda$ is maximized at the configuration $\omega_{N}$ that minimizes $\mathscr{H}\left(\omega_{N}\right)$. The optimum arrangements $\left\{x_{1}, \ldots, x_{N}\right\}$ that minimize $\mathscr{H}\left(\omega_{N}\right)$ are numerically computed by different methods.

\section{Dissipative Operators}

I restrict myself to definitions (cf. Pazy [19]). Let $X$ be a Banach space whose norm is denoted by $\|\cdot\|, X^{*}$ its dual, and $x^{*} \in X^{*}$. The value of $x^{*}$ at $x \in X$ is denoted by $\left\langle x^{*}, x\right\rangle=\left\langle x, x^{*}\right\rangle=x^{*}(x)$.

Definition 18. For $x \in X$, we define the duality set by

$$
F(x)=\left\{x^{*} \in X^{*} ; x^{*}(x)=\|x\|^{2}=\left\|x^{*}\right\|^{2}\right\} .
$$

Definition 19. A linear operator $A$, in $X$, with domain $D(A)$, is dissipative if, for every $x \in D(A)$, there exists $x^{*} \in F(x)$ such that $\Re\left\langle A x, x^{*}\right\rangle \leq 0$ or, equivalently, if $\|(\lambda I-A) x\| \geq \lambda\|x\|$ for all $x \in D(A)$ and $\lambda>0$. A dissipative operator $A$ is called $m$-dissipative if, for all $\lambda>0$, the operator $\lambda I-A: D(A) \rightarrow X$ is surjective.

The Lumer-Phillips Theorem states that a densely defined operator $A$ in $X$ is the generator of a $C_{o}$-semigroup $T(t)$ (i.e., $\lim _{t \rightarrow 0} T(t) x=x$ ) of contractions if and only if it is $m$ dissipative (cf. [19]). I recall that the domain $D(A)$ of the infinitesimal generator $A$ of $T(t)$ is

$$
D(A)=\left\{x \in X: \lim _{t \rightarrow 0} \frac{T x-x}{t} \text { exists }\right\}
$$

and, for any $x \in D(A)$,

$$
A x=\lim _{t \rightarrow 0} \frac{T x-x}{t} .
$$

\section{Dynamical Systems}

In this section, I restrict myself to several definitions and examples of dynamical systems and also definitions and some properties of limit and invariant sets. More investigations into this large topic are in preparation.

Different definitions of dynamical systems are given in the literature.

\subsection{Definitions}

7.1.1. General Definitions. In (2011), Kloeden and Rasmussen give, in [20], the general definitions:

let $\mathbb{T}=\mathbb{R}[$ resp., $\mathbb{Z}], \mathbb{\mathbb { T }}_{o}^{+}=\mathbb{\mathbb { V }} \cap[0, \infty)$, that is, $\mathbb{R}^{+}$[resp., $\mathbb{N}]$ and a metric space $X$.

Definition 20. A dynamical system is a continuous map $\Phi$ : $\mathbb{T} \times X \rightarrow X$ which satisfies the initial value condition

$$
\Phi(0, x)=x \quad \forall x \in X
$$

and the group property

$$
\Phi(s+t, x)=\Phi(s, \Phi(t, x)) \quad \forall s, t \in \mathbb{T}, x \in X .
$$

When $\mathbb{T}=\mathbb{R}$ the dynamical system is called continuous and when $\mathbb{T}=\mathbb{Z}$ the dynamical system is called discrete.

Definition 21. A semidynamical system is a continuous map $\Phi: \mathbb{T}_{o}^{+} \times X \rightarrow X$ which satisfies the initial value condition

$$
\Phi(0, x)=x \quad \forall x \in X
$$

and the semigroup property

$$
\Phi(s+t, x)=\Phi(s, \Phi(t, x)) \quad \forall s, t \in \mathbb{T}_{o}^{+}, x \in X
$$

When $\mathbb{T}_{o}^{+}=\mathbb{R}^{+}$[resp., $\left.\mathbb{N}\right]$ the semidynamical system is called continuous [resp., discrete].

7.1.2. "Classical” Dynamical Systems. Let B be a Banach space. In 1969, Hale [21] gives the following definition.

Definition 22. A classical dynamical system on $B$ is a continuous function $u: \mathbb{R}^{+} \times B \rightarrow B$ which satisfies

$$
u(0, \varphi)=\varphi, \quad u(t+\tau, \varphi)=u(t, u(\tau, \varphi))
$$

for all $t, \tau \geq 0$ and $\varphi \in B$.

Remark 23. In Definition 22, the mapping $u$ is a continuous semidynamical system in terms of Definition 21.

7.1.3. "Abstract" Dynamical System. In 1991, Haraux [22] defines abstract dynamical systems in a complete metric space $(E, d)$ in the following way.

Definition 24. An abstract dynamical system in $E$ is a family $\{S(t)\}_{t \geq 0}$ of mappings on $E$ which satisfy the following properties:

(i) for each $t \geq 0, S(t): E \rightarrow E$ is continuous,

(ii) $S(0)=I$,

(iii) $S(t+s)=S(t) S(s)$,

(iv) for each $z \in E, t \in[0, \infty) \rightarrow S(t) z \in E$ is continuous.

Remark 25. In Definition 24, the mapping $u:(t, z) \in \mathbb{R}^{+} \times$ $E \rightarrow u(t, z)=S(t) z \in E$ satisfies conditions (45) and (46) but it is only separately continuous. Therefore it is not a semidynamical system in terms of Definition 21 . 
7.1.4. Dafermos Definition. In [23, Definition 4.1], Dafermos gives the following definition in a metric space $X$.

Definition 26. A Dafermos dynamical system in $X$ is a map $u: X \times \mathbb{R}^{+} \rightarrow X$ which satisfies the initial condition

$$
u(x, 0)=0 \quad \forall x \in X
$$

the semigroup property

$$
u(x, s+t)=u(u(x, s), t) \quad \forall t, s \in \mathbb{R}^{+}, x \in X,
$$

and the continuity condition in $x$, for all $t \in \mathbb{R}^{+}$.

Remark 27. In the previous definitions the space $X$ [resp. $B$, $E]$ is called the phase space, and the set

$$
\gamma^{+}(x)=\bigcup_{t \geq 0} \Phi(t, x)\left[\operatorname{resp} . \bigcup_{t \geq 0} u(t, x)\right]
$$

is called the positive orbit or trajectory through $x \in X$ [resp., $x \in B, x \in E]$.

\subsection{Examples}

\subsubsection{Continuous (Semi-)Dynamical Systems}

Example 28 (cf. [21, Example 1]). Let $f$ be a continuous function $\mathbb{R}^{n} \rightarrow \mathbb{R}^{n}$ and $\xi \in \mathbb{R}^{n}$. Consider the autonomous differential equation

$$
\frac{d x}{d t}=f(x(t)), \quad t \geq 0,
$$

with the initial condition

$$
x(0)=\xi
$$

If there exists a unique solution $u(t, \xi)$ of (51) and (52) which depends continuously upon $t, \xi$, then the mapping

$$
\Phi: \mathbb{R}^{+} \times \mathbb{R}^{n} \longrightarrow \mathbb{R}^{n} \quad \text { defined by } \Phi(t, \xi)=u(t, \xi)
$$

is a continuous semidynamical system on $\mathbb{R}^{n}$ (Definitions 21 and 22).

Example 29 (cf. [20, Example 1.6]). Let $X$ be the Banach space of bounded continuous functions $\mu:[-1,0] \rightarrow \mathbb{R}^{d}$, under the usual norm $\|\cdot\|_{\infty}$. Let $\mu \in X$ and $f$ a continuous function $\mathbb{R}^{d} \rightarrow \mathbb{R}^{d}$ and suppose that there exists a unique solution $u(t, \mu)$ of the autonomous delay differential equation

$$
\frac{d x}{d t}=f(x(t-1)), \quad t \geq 0,
$$

with the condition

$$
x(s)=\mu(s), \quad s \in[-1,0] .
$$

Then

$$
\begin{aligned}
\Phi:(t, \mu) \in \mathbb{R}^{+} \times X \longrightarrow \Phi(t, \mu)(s) & =u(t+s, \mu) \in X, \\
t & \geq 0, s \in[0,1],
\end{aligned}
$$

is a continuous semidynamical system on $X$.
Example 30 (see cf. [22, page 12]). Let $X$ be a real Banach space, $A$ a $m$-dissipative operator with dense domain in $X$ (cf. Section 5$), T(t)$ the semigroup of contractions generated by $A$, and $F: X \rightarrow X$ a Lipschitzian function on the bounded sets of $X$. Let $u(t, x) \in C([o, \tau(x)), X)$ be the unique maximal solution of

$$
\begin{array}{r}
u(t, x)=T(t) x+\int_{o}^{t} T(t-s) F(u(s)) d s, \\
t \in[0, \tau(x)), x \in X .
\end{array}
$$

Set $u(t, x)=S(t) x$. Let $Y \subset X$ such that $\tau(y)=\infty$ for all $y \in$ $Y$ and $\|S(t) y\| \leq M$, for all $t \geq 0, y \in Y$. For $y \in Y, u(t, y)=$ $S(t) y$ is solution of (57) for $x=y$. Set $Z=\overline{\bigcup_{y \in Y} \bigcup_{t \geq 0}\{S(t) y\}}$ and denote by $d$ the distance induced on $Z$ by the norm in $X$. Then $\{S(t)\}_{t \geq 0}$ is an abstract dynamical system (Definition 24) on $(Z, d)$.

Example 31. Dafermos dynamical systems are adapted to equations of linear viscoelasticity. See Section 10.

Example 32 (see cf. [20]). Let $X$ be a metric space and $f$ : $X \rightarrow X$ be a continuous function. By iteration, $f^{n+1}(x)=$ $f\left(f^{n}\right)(x)=f o f^{n}(x)$, for all $x \in X, n \in N$, is well defined. The mapping

$$
\Phi:(n, x) \in \mathbb{N} \times X \longrightarrow \Phi(n, x)=f^{n}(x) \in X
$$

is a discrete semidynamical system (Definition 21). Now, suppose $f$ is an homeomorphism; that is, it is continuous and invertible with continuous inverse. Then, the mapping $\Phi$ defined by (58) can be extended to $\mathbb{Z}^{-}$by

$$
\Phi(n, x)=\left(f^{-1}\right)^{-n}(x), \quad \forall n \in \mathbb{Z}^{-}, x \in X,
$$

and $\Phi$ is a discrete dynamical system.

7.2.2. Discrete-Time Dynamical Systems and Fractals. What is known as a discrete time dynamical system in Pesin and Climenhaga [24] is related to a map from a set to itself. Let $X$ be any set and $f$ a map $f: X \rightarrow X$. By iteration, $f^{n+1}(x)=$ $f\left(f^{n}(x)\right)=\left(f \circ f^{n}\right)(x)$ is well defined for every $n \in \mathbb{N}$ and we have the semigroup property:

$$
f^{n+m}=f^{m} \circ f^{n}=f^{n} \circ f^{m}
$$

for any integer $m, n \geq 0$.

Definition 33. Let $x \in X$. The sequence of points $f^{o}(x)=$ $x, f(x), f^{2}(x), \ldots$, is called the trajectory or orbit of $x$.

\subsubsection{Examples with Chaotic Behavior}

Example 34 (the Cantor set). Consider, in $\mathbb{R}$, the intervals $I_{1}=[0,1 / 3], I_{2}=[2 / 3,1]$, and $D=I_{1} \cup I_{2}$ and the map $f$ defined by $f(x)=3 x$ on $I_{1}$ and $3 x-2$ on $I_{2}$. The range of $f$ is $[0,1]$ and does not lie in its domain of definition. The domain of definition of $f^{2}$ is $\{x ; x \in D$ and $f(x) \in D\}$, that is, the union of the intervals:

$$
\left[0, \frac{1}{9}\right], \quad\left[\frac{2}{9}, \frac{1}{3}\right], \quad\left[\frac{2}{3}, \frac{7}{9}\right], \quad\left[\frac{8}{9}, 1\right]
$$


Figure 1: The Cantor set.

obtained by removing the open middle third of each interval $I_{1}$ and $I_{2}$ and so on. The domain on which every iterate $f^{n}$ is defined is exactly the Cantor set (cf. Figure 1).

Example 35 (the Sierpinski triangle (or gasket)). An equilateral triangle is divided into four smaller triangles, each similar to the first and congruent to each other. Then, the middle triangle is removed, and the iterating procedure is iterated on the remaining three, and so on. The fractal $T$ obtained as the limit of this procedure is the Sierpinki triangle (cf. Figure 2). With this procedure is associated the following algorithm. If $x \in T$, and only in this case, it is possible to define a function $x \in T \rightarrow f(x) \in \mathbb{R}^{2}$ such that $f^{n}(x) \in T$ for every $n$ (cf. Schroeder [25] which calls this procedure "Sir Pinski game").

The Cantor set and the Sierpinski triangle are examples of fractals (cf. $[26,27])$.

\subsection{Limit and Invariant Sets}

Definition 36 (see cf. [20]). Let $\Phi: \mathbb{T} \times X \rightarrow X$ be a dynamical system in a metric space $X$. The $\omega$-limit set [resp., $\alpha$-limit set] of a point $\xi \in X$ is defined by

$$
\begin{gathered}
\omega(\xi)=\bigcap_{\tau \geq 0} \overline{\bigcup_{t \geq \tau} \phi(t, \xi)} \\
{\left[\text { resp., } \alpha(\xi)=\bigcap_{\tau \leq 0} \overline{\bigcup_{t \leq \tau} \phi(t, \xi)}\right] .}
\end{gathered}
$$

In the case of a semidynamical system $\left(\Phi: T_{o}^{+} \times X \rightarrow X\right)$ the notion of a $\alpha$-limit set is not defined. In the same way, $\omega$ and $\alpha$-limit sets of a subspace $M \subset X$ are defined by (62) where $\xi$ is replaced by $M$.

Remark 37. The $\omega$ and $\alpha$-limit sets may be defined as follows:

$\omega(\xi)=\left\{x \in X:\right.$ there exists a sequence $t_{n}, t_{n}>0$,

$$
\left.t_{n} \longrightarrow \infty \text { such that } \Phi\left(t_{n}, \xi\right) \longrightarrow x \text { as } n \longrightarrow \infty\right\}
$$

$\alpha(\xi)=\left\{x \in X:\right.$ there exists a sequence $t_{n}, t_{n}<0$,

$$
\left.t_{n} \longrightarrow-\infty \text { such that } \Phi\left(t_{n}, \xi\right) \longrightarrow x \text { as } n \longrightarrow \infty\right\} \text {. }
$$

Remark 38. Similar definitions are valid for classical and abstract dynamical systems.

Definition 39. Let $\Phi: \mathbb{T}_{o}^{+} \times X \rightarrow X$ be a semidynamical system on the metric space $X$ (Definition 21). A subset $M \subset X$ is called invariant under $\Phi$ if

$$
\Phi(t, M)=M \quad \forall t \in \mathbb{T}_{o}^{+} .
$$

It is called positively [resp., negatively] invariant if

$$
\Phi(t, M) \subset M \quad[\text { resp., } M \subset \Phi(t, M)], \quad \forall t \in \mathbb{T}_{o}^{+} .
$$

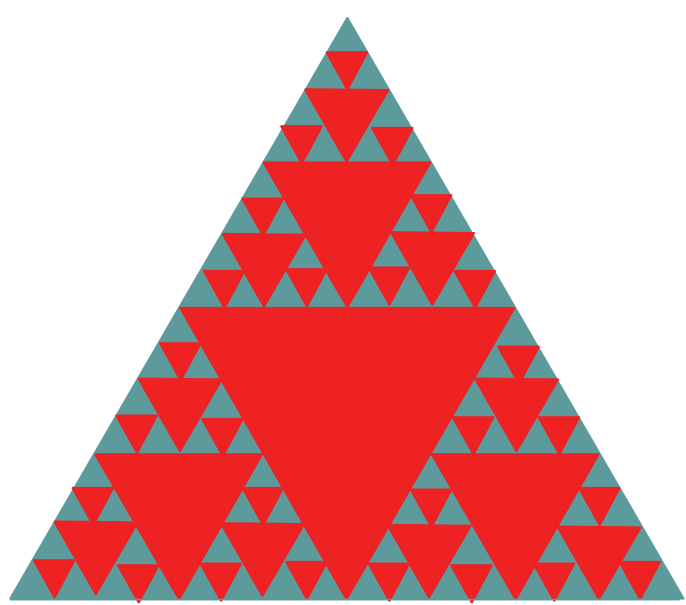

FIGURE 2: The Sierpinski gasket.

In the case of discrete-time dynamical system and of Example 32, invariance is equivalent to $f(M)=M$.

Properties of $\omega$-Limit and Invariant Sets. Let $(X, d)$ be a metric space, $\Phi: \mathbb{\mathbb { T }}_{o}^{+} \times X \rightarrow X$ a semidynamical system on $X$, and $x \in X$. If the trajectory $\gamma^{+}(x)$ through $x$ is precompact, then $\omega(x)$ has the following properties:

$$
\Phi(t, \omega(x))=\omega(x) \neq \emptyset, \quad \forall t \in \mathbb{T}_{o}^{+},
$$$$
\omega(x) \text { is a compact subset of } X \text {, }
$$

$$
\omega(x) \text { is connected if } \mathbb{T}_{o}^{+}=\mathbb{R}^{+} \text {. }
$$

If $\mathbb{T}_{o}^{+}=\mathbb{N}, \omega(x)$ does not need to be connected (cf. [20, Exercise 1.10]).

In the case of an abstract dynamical system $\left\{(S(t)\}_{t \geq 0}\right.$, on a complete metric space $(E, d)$, previous results hold with $\Phi(t, x)=S(t) x$, for $x \in E$, if $\gamma^{+}(x)$ is relatively compact in $E$ (see [28, page 122]). But in a complete metric space $E$ a subset $A$ is relatively compact if and only if it is precompact (cf. [29]).

\section{Equal-Area Condition}

Equal-area type conditions appear, as sufficient or necessary conditions, in the formation of layers (internal or superficial) in stationary solutions to various singularly perturbed reaction-diffusion systems. In the recent works do Nascimento [30], Crema and do Nascimento [31], and do Nascimento and de Moura [32], the authors prove the necessity of suitable equal-area condition for the formation of internal or (and) superficial transition layers in this type of problems.

Example 40. A simple particular case of problems studied in [30] is the elliptic boundary value problem

$$
\begin{gathered}
\epsilon \operatorname{div}(h(x) \nabla u)+f(u)=0, \quad x \in \Omega, \\
\frac{\partial u}{\partial n}=0 \quad \text { on } \partial \Omega,
\end{gathered}
$$


where $\Omega$ is a smooth domain in $\mathbb{R}^{N}, N \geq 1, f: \mathbb{R} \rightarrow \mathbb{R}$ such that there exist $\alpha, \beta, \alpha>\beta$, with $f(\alpha)=f(\beta)=0$.

Let $\Gamma \subset \Omega$ be a smooth $(N-1)$-dimensional compact manifold without boundary. It is proved that, if (67) has a family $\left\{u_{\epsilon}\right\}$ of solutions which develop an internal transition layer with interface $\Gamma$ connecting the states $\alpha$ to $\beta$, then, necessarily, the simple equal-area condition

$$
\int_{\alpha}^{\beta} f(s) d s=0
$$

is satisfied.

Example 41. In [31], the following stationary system is considered:

$$
\begin{gathered}
\epsilon \operatorname{div}(h(x) \nabla u)+f(x, u, \mathbf{v})=0, \quad x \in \Omega, \\
\operatorname{div}(\mathbf{k}(x) \nabla \mathbf{v})+\mathbf{g}(x, u, \mathbf{v})=0, \quad x \in \Omega, \\
\frac{\partial \mathbf{v}}{\partial n}=0 \quad(\text { or } \mathbf{v}=0) \text { on } \partial \Omega,
\end{gathered}
$$

where $\Omega$ is a smooth domain in $\mathbb{R}^{N}, N \geq 1, f \in C^{1}(\Omega \times \mathbb{R} \times$ $\left.\mathbb{R}^{N}\right), \mathbf{v}, \mathbf{k}$, and $\mathbf{g}$ are sufficiently smooth $\mathbb{R}^{n}$-valued functions, and $\mathbf{k} \nabla \mathbf{v}=\left(k_{1} \nabla v_{1}, \ldots, k_{n} \nabla v_{n}\right)$.

Let $\mathcal{U}$ be an open connected set in $\Omega, \Gamma \subset \overline{\mathcal{U}}$ be an $(N-1)$ dimensional compact connected orientable manifold whose boundary $\partial \Gamma$ is such that $\partial \Gamma \cap \partial \Omega$ is an $(N-2)$-dimensional submanifold of $\partial \Omega$. The authors give a definition of a family of internal transition layer solutions $\left.\left\{\left(u_{\epsilon}, \mathbf{v}_{\epsilon}\right)\right\}, 0<\epsilon<\epsilon_{o}\right\}$ to (69) in $\mathcal{U}$ with interface $\Gamma$, depending on two functions $\alpha, \beta \in$ $C^{o}(\mathcal{U}), \alpha(x)<\beta(x)$ on $\Gamma$. They show that, for such a family, there exists $u_{o}\left[\right.$ resp., $\mathbf{v}_{o}$ ] such that $u_{\epsilon} \rightarrow u_{o}$ [resp., $\mathbf{v}_{\epsilon} \rightarrow \mathbf{v}_{o}$ ] on compact sets of $\overline{\mathscr{U}} \backslash \Gamma$ [resp., in $\overline{\mathscr{U}}]$. They also prove that, if a family of internal transition layer solutions to (69) exists, then $f\left(x, u_{o}(x), \mathbf{v}_{o}(x)\right)=0$ on $\mathcal{U} \backslash \Gamma$ and necessarily the equal-area condition

$$
\int_{\Gamma}\left(\int_{\alpha(x)}^{\beta(x)} f\left(x, s, \mathbf{v}_{o}(x)\right) d s\right) d S=0
$$

is satisfied. Several concrete applications of these results are presented in the paper.

Example 42. In [32], $\Omega$ is a bounded domain in $\mathbb{R}^{N}, N \geq 1$, with $C^{2}$ boundary $\partial \Omega, S$ is a $C^{2}(N-1)$-dimensional surface with a boundary $\Sigma$ which is assumed to be a $C^{2}(N-2)$ dimensional compact surface without boundary with $\Sigma=\bar{S} \cap$ $\partial \Omega$, and $\bar{S}$ intersects $\partial \Omega$ transversally. The authors define a family of solutions $\left\{u_{\epsilon}\right\}, 0<\epsilon<\epsilon_{o}$, to the elliptic boundary value problem:

$$
\begin{gathered}
\epsilon \operatorname{div}\left(a(x) \nabla v_{\epsilon}\right)+f\left(x, v_{\epsilon}\right)=0, \quad x \in \Omega, \\
\epsilon a(x) \frac{\partial v_{\epsilon}}{\partial \widehat{n}}=g\left(x, v_{\epsilon}\right), \quad x \in \partial \Omega,
\end{gathered}
$$

which develops internal and superficial transition layers, depending on some smooth functions $\alpha, \beta, \alpha(x)<\beta(x)$, $x \in \bar{\Omega}$, with interfaces $S$ and $\Sigma$, respectively.
Here $a \in C^{1}(\bar{\Omega}), a>0, f: \Omega \times \mathbb{R} \rightarrow \mathbb{R}$, and $g: \partial \Omega \times \mathbb{R} \rightarrow$ $\mathbb{R}$ are of class $C^{1}$ and $\widehat{n}$ is the exterior normal vector field on $\partial \Omega$. It is proved that the equal-area conditions

$$
\begin{array}{ll}
\int_{\alpha(x)}^{\beta(x)} f(x, t) d t=0 & \forall x \in S, \\
\int_{\alpha(y)}^{\beta(y)} g(y, t) d t=0 & \forall y \in \Sigma
\end{array}
$$

are necessary for the existence of such solutions.

\section{Inertial Manifolds}

9.1. Setup. Let $H$ be a Hilbert space and $S(t)$ the semigroup associated with an evolution equation of the form

$$
u_{t}^{\prime}+A u+R u=0
$$

with the initial condition $u(0)=u_{o} \in H$, where $A$ is a linear operator and $R$ a nonlinear one. When an inertial manifold $M$ exists for problem (73), the restriction of (73) to $M$ reduces to a finite dimensional ordinary differential equation (80), which is an exact copy of the initial system (cf. [33]). The manifold $M$ is usually viewed as the graph of a suitable smooth function $\Phi: P H \rightarrow Q H$, where $P$ is an orthogonal projection on $H$ with finite-dimensional range, and $Q=I-P$.

After some definitions, the following sections will be devoted to the existence of an inertial manifold for (73) and the presentation of results on the existence and the behavior of inertial manifolds for phase-field equations.

9.2. Definitions. Let $(E, d)$ be a metric space and $S(t)$ a continuous semigroup on $E$.

Definition 43. A set $M \subset E$ is an inertial manifold for $S(t)$ if

(i) $M$ is a finite-dimensional Lipschitz manifold in $E$,

(ii) $M$ is of class $C^{1}$,

(iii) $M$ is positively invariant under the flow that is, $S(t) M \subset M$, for all $t \geq 0$,

(iv) $M$ is exponentially attracting; that is, there exists a constant $c_{o}$ such that, for any $u_{o} \in E$, there exists a constant $c_{1}\left(u_{o}\right)>0$ such that

$$
\operatorname{dist}_{E}\left(S(t) u_{o}, M\right) \leq c_{1} e^{-c_{o} t}, \quad \forall t \geq 0,
$$

where dist ${ }_{E}$ is the Hausdorff semidistance (see Section 1.8 in Huet [7]); compare Bonfoh et al. [34, page 164].

Definition 44 (see Temam [33]). Let $B \subset E$ and $U$ an open subset of $E$ such that $B \subset U ; B$ is said to be absorbing for $S(t)$ in $U$ if, for any bounded set $B_{o}$ in $U$, there exists $t_{1}\left(B_{o}\right)$ such that $S(t) B_{o} \subset B$, for all $t \geq t_{1}$. In particular, if $B$ is bounded, $S(t) B \subset B$, for $t \geq t_{1}(B)$.

Remark 45. Similar definitions were given in [33] and Luskin and Sell [35], in the case of Hilbert spaces. In [33] the smoothness of $M$ and the continuity of the semigroup $S(t)$ are not parts of the definition of an inertial manifold. 
9.3. Existence of an Inertial Manifold for Problem (73) (cf. [33]). In a Hilbert space $H$ whose norm is denoted by $|\cdot|$, consider problem (73) where $A$ is an unbounded linear operator in $H$, strictly positive (i.e., $\exists c>0$ such that $(A u, u) \geq c\|u\|_{H}^{2}$ for all $u$ in the domain $D(A)$ of $\left.A\right)$, and self-adjoint. It is assumed that $A^{-1}$ is compact. Thanks to assumptions on $A$, it is possible to define its powers $A^{s}$ defined on $D\left(A^{s}\right)$, for all $s \in \mathbb{R}$. When $0<s<1$ see also Huet [36], Kato [37], Schechter [38]. For $r>0$, we denote by $B_{s, r}$ the ball, in $D\left(A^{s}\right)$, with center 0 of radius $r$; that is,

$$
B_{s, r}=\left\{v \in D\left(A^{s}\right) ;\|v\|_{s}=\left|A^{s} v\right| \leq r\right\} .
$$

It is assumed that $R$ satisfies the following properties.

$\mathrm{H}_{1}$. There exists $\alpha \in \mathbb{R}$ such that $R$ is Lipschitz on the bounded sets of $D\left(A^{\alpha}\right)$ with values in $D\left(A^{\alpha-1 / 2}\right)$ and

$$
\left|A^{\alpha-1 / 2} R(u)-A^{\alpha-1 / 2} R(v)\right| \leq C_{K}\left|A^{\alpha}(u-v)\right|,
$$

$\forall u, v \in D\left(A^{\alpha}\right)$ satisfying $\left|A^{\alpha} u\right| \leq K, \quad\left|A^{\alpha} v\right| \leq K$.

$\mathrm{H}_{2}$. If $u_{o} \in D\left(A^{\alpha}\right)$ problem (73) has a unique solution $u \in C\left(\mathbb{R}^{+} ; D\left(A^{\alpha}\right)\right) \cap L^{2}\left((O, T) ; D\left(A^{\alpha+1 / 2}\right)\right)$, for all $T \geq 0$, and the map $S(t): u_{o} \rightarrow u(t)$ is continuous from $D\left(A^{\alpha}\right)$ into itself, for all $t \geq 0$.

$\mathrm{H}_{3}$. The semigroup $S(t)$ possesses an absorbing set $B_{o} \subset$ $D\left(A^{\alpha}\right)$ such that

(1) $S(t) B_{o} \subset B_{o}$, for all $t \geq O$,

(2) the $\omega$-limit set of $B_{o}$

$$
\omega\left(B_{o}\right)=\bigcap_{\beta \geq 0} \overline{\substack{t \geq \beta \\ t \geq \beta}}
$$

is the maximal attractor for $S(t)$ in $D\left(A^{\alpha}\right)$.

(3) $\rho>0$ is chosen such that $B_{o}$ is included in the ball $B_{\alpha, \rho / 2}$ of $D\left(A^{\alpha}\right)$ (cf. (75)).

9.3.1. The Prepared Equation. The prepared equation is equivalent to the original one for $t$ large. Let $\theta: \mathbb{R}^{+} \rightarrow[0,1]$ be a $C^{\infty}$ function such that

$$
\begin{gathered}
\theta(s)= \begin{cases}1 \quad \text { for } 0 \leq s \leq 1, \\
0 \quad \text { for } s \geq 2,\end{cases} \\
\sup _{s \geq 0}\left|\theta^{\prime}(s)\right| \leq 2 .
\end{gathered}
$$

The aim of the prepared equation is to avoid the difficulties related to the behavior of the nonlinear term $R(u)$ for large values of $\left|A^{\alpha}(u)\right|$. Let $\theta_{r}(s)=\theta(s / r), r>0$, and

$$
R_{r}(u)=\theta_{r}\left(\left|A^{\alpha}(u)\right|\right) R(u) \quad \forall u \in D\left(A^{\alpha}\right) .
$$

The prepared equation associated with (73) is of the form

$$
u_{t}^{\prime}+A u+R_{\rho} u=0
$$

where $\rho$ is chosen in $\mathrm{H}_{3}(3)$. Let $S_{\rho}(t)$ be the semigroup associated with (80). The following is assumed.
$\mathrm{H}_{4}$. The ball $B_{\alpha, \rho}$ is absorbing for $S_{\rho}(t)$.

Thanks to the assumptions on $R, R_{\rho}$ is a bounded operator and a Lipschitz mapping from $D\left(A^{\alpha}\right)$ into $D\left(A^{\alpha-1 / 2}\right)$. In particular

$$
\begin{array}{r}
\left|R_{\rho}\left(u_{1}\right)-R_{\rho}\left(u_{2}\right)\right| \leq C_{\rho}\left|A^{\alpha}\left(u_{1}-u_{2}\right)\right| \\
\forall u_{1}, u_{2} \in D\left(A^{\alpha}\right),
\end{array}
$$

where $C_{\rho}$ is a constant which depends on $\rho$.

9.3.2. Space $\mathscr{F}_{N b l}^{\alpha}$. Let $N \in \mathbb{N}, b, l>0$ and $\alpha$ defined in $H_{1}$. Under the assumptions of $A$, there exists an orthonormal basis $w_{j}$ in $H$, where $w_{j}$ is the eigenvector of $A$ corresponding to the eigenvalue $\lambda_{j}$, with $0<\lambda_{1} \leq \lambda_{2} \cdots \leq \lambda_{j} \rightarrow \infty$, as $j \rightarrow \infty$. Set

$$
P=P_{N} \quad Q=I-P=I-P_{N},
$$

where $P_{N}$ is the orthogonal projector, in $H$, onto the space spanned by $w_{1}, \ldots w_{N}$. The projections $P$ and $Q$ commute with $A^{\beta}$, for all $\beta \in \mathbb{R}$.

Definition 46. Let $\mathscr{F}$ be the space defined by

$$
\mathscr{F}=\mathscr{F}_{N b l}^{\alpha}=\left\{\varphi: P D\left(A^{\alpha}\right) \longrightarrow Q D\left(A^{\alpha}\right)\right\},
$$

where $\varphi$ is a Lipschitz function with $\operatorname{supp}(\varphi) \subset\{p \in$ $\left.P D\left(A^{\alpha}\right),\left|A^{\alpha} p\right| \leq 2 \rho\right\}$, that satisfies

$$
\begin{gathered}
\left|A^{\alpha} \varphi(p)\right| \leq b \\
\left|A^{\alpha} \varphi\left(p_{1}\right)-A^{\alpha} \varphi\left(p_{2}\right)\right| \leq l\left|A^{\alpha}\left(p_{1}-p_{2}\right)\right|
\end{gathered}
$$

for all $p \in P D\left(A^{\alpha}\right), p_{1}, p_{2} \in D\left(A^{\alpha}\right)$.

Remark 47. (1) $P D\left(A^{\alpha}\right)$ is finite dimensional.

(2) $\mathscr{F}$ is a complete metric space for the distance

$$
d\left(\varphi_{1}, \varphi_{2}\right)=\sup _{p \in P D\left(A^{\alpha}\right)}\left|A^{\alpha}\left(\varphi_{1}(p)-\varphi_{2}(p)\right)\right| .
$$

9.3.3. Construction of a Map $\mathscr{T}: \mathscr{F} \rightarrow \mathscr{F}$. Let $\varphi \in \mathscr{F}, p_{o} \in$ $P D\left(A^{\alpha}\right)$. Thanks to assumptions on $\varphi$ and $R_{\rho}$, the problem

$$
\frac{d p}{d t}+A p+P R_{\rho}(p+\varphi(p))=0, \quad p(0)=p_{o}
$$

has a unique solution $p_{p_{o}, \varphi}(t) \in P D\left(A^{\alpha}\right)$. Then, the problem

$$
\frac{d q}{d t}+A q=-Q R_{\rho}\left(p_{p_{o}, \varphi}(t)+\varphi\left(p_{p_{o}, \varphi}(t)\right)\right)
$$

has a unique solution $q_{p_{o}, \varphi}(t) \in C_{b}\left(\mathbb{R} ; Q D\left(A^{\alpha}\right)\right)$. Therefore $q_{p_{0}, \varphi}(0) \in \mathrm{Q} D\left(A^{\alpha}\right)$ (cf. Lemma 2.3, page 420 in [33]). The mapping $\mathscr{T}$ is defined by $\psi=\mathscr{T}(\varphi)$ with

$$
\psi: p_{o} \in P D\left(A^{\alpha}\right) \longrightarrow q_{p_{o}, \varphi}(0) \in Q D\left(A^{\alpha}\right) .
$$

Let $\sigma(t)$ denote the right-hand side of (87). Then

$$
\psi\left(p_{o}\right)=-\int_{-\infty}^{0} e^{t A} \sigma(\tau) d \tau
$$


9.3.4. Existence of an Inertial Manifold for (73) or (80). Under assumptions $H_{1}-H_{4}$, if it is possible to find $N, b$, and $l$, such that $\mathscr{T}$ is a strict contraction of $\mathscr{F}$ into itself, that is, $\exists L<1$ with $d\left(\mathscr{T} \varphi_{1}, \mathscr{T} \varphi_{2}\right) \leq L d\left(\varphi_{1}, \varphi_{2}\right)$, for all $\varphi_{1}, \varphi_{2} \in \mathscr{F}$, then, $\mathscr{T}$ has a fixed point $\Phi \in \mathscr{F}$, and the graph of $\Phi$ is an inertial manifold for (73) or (80).

\subsection{Inertial Manifolds for Phase-Field Equations}

Example 48 (cf. Bonfoh [39]). Set $\Omega=\prod_{i=1}^{n}\left(0, L_{i}\right), L_{i}>0$, and $n \leq 2$ and denote by $N$ the operator $-\Delta: H^{2}(\Omega) \cap$ $H_{o}^{1}(\Omega) \rightarrow L^{2}(\Omega)$. Consider the phase-field equations $\left(P_{\epsilon}\right)$,

$$
\begin{gathered}
\delta \phi_{t}+N \phi+g(\phi)=u, \\
\epsilon u_{t}+\phi_{t}+N u=0
\end{gathered}
$$

in $\Omega$, with the boundary conditions

$$
\phi,\left.u\right|_{\partial \Omega}=0
$$

and the initial conditions

$$
\left.\phi\right|_{t=0}=\phi_{o},\left.\quad u\right|_{t=0}=u_{o}
$$

where $\delta>0, \epsilon \in\left(0, \epsilon_{o}\right.$, , and $g \in C^{2}(\mathbb{R})$ satisfies the conditions

$$
\begin{gathered}
\left|g^{\prime}(s)\right| \leq C_{1}\left(|s|^{p}+1\right), \quad g^{\prime}(s) \geq-C_{2} \\
C_{1}>0, C_{2} \geq 0, \quad p>0, \quad \forall s \in \mathbb{R} .
\end{gathered}
$$

The limit problem $\left(P_{o}\right)$ is given by $(90)$ where $\epsilon=0$ :

$$
\begin{gathered}
\delta \phi_{t}+N \phi+g(\phi)=u, \\
\phi_{t}+N u=0
\end{gathered}
$$

the boundary conditions (91), and the initial condition $\left.\phi\right|_{t=0}=$ $\phi_{o}$. Set

$$
\begin{aligned}
& H_{1}=H_{o}^{1}(\Omega), \quad H_{2}=H^{2}(\Omega) \cap H_{o}^{1}(\Omega), \\
& H_{3}=\left\{q \in H_{3}(\Omega) \cap H_{o}^{1}(\Omega) ; \Delta q \in H_{o}^{1}(\Omega)\right\} .
\end{aligned}
$$

Let $\|\cdot\|$ denote the usual norm in $L^{2}(\Omega)$ and set $\|q\|_{r}=$ $\left\|N^{r / 2} q\right\|, r=1,2,3$. The norms $\|\cdot\|_{r}$ are equivalent to the usual Sobolev $H^{r}(\Omega)$ norms on $H_{r}$. Several Banach spaces whose norms depend on $\epsilon$ are introduced. In particular

$$
\mathscr{H}_{\epsilon}^{o}=H_{1} \times L^{2}(\Omega) \text {, }
$$

with the norm $\|(p, q)\|_{\mathscr{H}_{\epsilon}^{o}}=\left(\|p\|_{1}^{2}+\epsilon\|q\|^{2}\right)^{1 / 2}$.

Under additional assumptions on the regularity of the data, there exist semigroups $S_{\epsilon}(t)$, global attractors $\mathscr{A}_{\epsilon}$, and exponential attractors $\mathscr{M}_{\epsilon}$ for problems $\left(P_{\epsilon}\right), 0 \leq \epsilon \leq \epsilon_{o}$ (cf. Chepyzhov and Vishik [40]). It is proved that $\mathscr{A}_{\epsilon}$ and $\mathscr{M}_{\epsilon}$ converge as $\epsilon \rightarrow 0$, to some lifting of $\mathscr{A}_{o}$ and $\mathscr{M}_{o}$, in $\mathscr{H}_{1}^{o}$ (cf. (96)). Moreover, there exists $r>0$, independent of $\epsilon$, such that

$$
\begin{gathered}
B=\left\{u \in H_{3},\|u\|_{3} \leq r\right\}, \\
B_{\epsilon}=\left\{(\phi, u) \in \mathscr{H}_{\epsilon}^{2},\|(\phi, u)\|_{\mathscr{H}_{\epsilon}^{2}} \leq r\right\}
\end{gathered}
$$

are bounded absorbing sets for $S(t)$ in $H_{3}$ and $S_{\epsilon}(t)$ in $\mathscr{H}_{\epsilon}^{2}$, respectively, where

$$
\mathscr{H}_{\epsilon}^{2}=H_{3} \times H_{2} \quad \text { with the norm }\|(p, q)\|_{\mathscr{H}_{\epsilon}^{2}}^{2}=\|p\|_{3}^{2}+\epsilon\|q\|_{2}^{2}
$$

and there exists $t_{1}>0$ such that $S(t) B \subset B, S_{\epsilon}(t) B_{\epsilon} \subset B_{\epsilon}$, for $t \geq t_{1}$. In particular

$$
\widetilde{B}=S\left(t_{1}\right) B \subset B, \quad \widetilde{B}_{\epsilon}=S_{\epsilon}\left(t_{1}\right) B_{\epsilon} \subset B_{\epsilon} .
$$

Following the classical method presented in the above section for (73), the authors show the existence of an inertial manifold $M^{r}$ to problem $\left(P_{o}\right)$ and of a family of inertial manifolds $M_{\epsilon}^{r}$ to problems $\left(P_{\epsilon}\right)$ such that

$$
M^{r} \subset \widetilde{B}, \quad M_{\epsilon}^{r} \subset \widetilde{B}_{\epsilon} .
$$

Let $\left(M^{r}\right)_{o}$ defined by

$$
\left(M^{r}\right)_{o}=\left\{\left(\phi,(I+\delta N)^{-1}(N \phi+g(\phi))\right), \phi \in M_{r}\right\} .
$$

Moreover, it is proved that

(1) for all $0<\epsilon_{2}<\epsilon_{1}$ small enough, $M_{\epsilon_{2}}^{r}$ is lower and upper semicontinuous at $\epsilon_{2}$, with respect to the metric induced by the $\mathscr{H}_{\epsilon_{1}}^{0}$ norm,

(2) $M_{\epsilon}^{r}$ converges, in a suitable sense, to $\left(M^{r}\right)_{o}$ with respect to the metric induced by the $\mathscr{H}_{1}^{0}$ norm, as $\epsilon$ goes to 0 .

\section{Mathieu-Hill Type Equations}

10.1. Mathieu and Hill Equations. The real Mathieu [resp., Hill] equation has the form

$$
y^{\prime \prime}+(a+b \cos 2 \pi x) y=0
$$

where $a$ and $b$ are constants,

$$
\left[\text { resp., } y^{\prime \prime}+(a+b q(x)) y=0\right]
$$

where $q$ is any smooth periodic function of period 1 with mean 0 (see Coddington and Levinson [41] and Wang and Guo [42]). Physical problems leading to Mathieu or Hill equations often require solutions with periodicity, called oscillatory solutions. Therefore, to find conditions on the data for which the above equations have a fundamental system of periodic solutions is a central problem. 
10.2. Its Equation. In [43], A. R. Its considers the Schrödinger equation on the positive semiaxis

$$
y^{\prime \prime}-\left[x^{\beta} p\left(x^{1+\alpha}\right)+c x^{-2}\right] y=0
$$

where $p$ is a smooth periodic function, with period 1 and mean $0, c$ is a real number, and the parameters $\alpha, \beta$ satisfy the relations $\beta-\alpha \geq-1$ and $2 \alpha-\beta>0$ (cf. Figure 3 ).

He proves that (104) has oscillatory solutions when $\beta>$ $\alpha-1$. If $\beta=\alpha-1$, the solutions are oscillatory or not. In all cases, asymptotic formulas for the solutions are stated, as $x \rightarrow \infty$. His method is based on a transformation which leads to a Hill-type equation and Floquet functions.

10.3. Method of Diagonalisation. In [44], Bodine and Lutz investigate (104) by a general method of diagonalization with a $L^{1}$-regularity for $p$. Set

$$
\begin{gathered}
t=x^{1+\alpha}, \quad \rho(t)=\int_{0}^{t} \frac{p(s)}{1+\alpha} d s \\
\mu=\int_{0}^{1} \rho(t) d t, \quad \nu=\int_{0}^{1}(\rho(t))^{2} d t \quad\left(\mu^{2}<\nu\right) .
\end{gathered}
$$

Let

$$
\begin{aligned}
& \lambda_{1}=\frac{1 / 2+\sqrt{c+\mu^{2}-v+1 / 4}}{1+\alpha}, \\
& \lambda_{2}=\frac{1 / 2-\sqrt{c+\mu^{2}-v+1 / 4}}{1+\alpha}
\end{aligned}
$$

be the eigenvalues of a suitable matrix which depends on $\alpha, c$, $\mu$, and $\nu$, and $\Lambda$ the diagonal matrix $\operatorname{diag}\left\{\lambda_{1}, \lambda_{2}\right\}$. The matrix $\Lambda$ is a crucial tool in the diagonalizable method used by the authors. When $\beta=\alpha-1$, three cases are studied separately as $\lambda_{1}-\lambda_{2}$ belongs to $\mathbb{N}$, or as it does not belong to $\mathbb{N}_{0}$ or as it is equal to 0 . In each case, very sharp asymptotic formulas are obtained for a fundamental system of solutions to (104), as $t \rightarrow \infty$. In particular, when $\lambda_{1}-\lambda_{2} \in \mathbb{N}_{o}$, a logarithmic term appears in the formulas. For instance, when $\lambda_{1}-\lambda_{2} \in \mathbb{N}$ the following formulas are obtained:

$$
\begin{aligned}
y_{1}(x)= & x^{\lambda_{1}(1+\alpha)}\left[1+\sum_{r=1}^{N-1} \frac{p_{r}^{1}\left(x^{1+\alpha}\right)}{x^{r(1+\alpha)}}+\mathcal{O}\left(\frac{1}{x^{N(1+\alpha)}}\right)\right] \\
& +\eta(\ln x) y_{2}(x), \\
y_{2}(x)= & x^{\lambda_{2}(1+\alpha)}\left[1+\sum_{r=1}^{N-1} \frac{p_{r}^{2}\left(x^{1+\alpha}\right)}{x^{r(1+\alpha)}}+\mathcal{O}\left(\frac{1}{x^{N(1+\alpha)}}\right)\right],
\end{aligned}
$$

where $p_{r}^{1,2}$ are scalar valued, bounded, continuous, periodic functions with period 1 which are recursively calculated and $\eta \neq 0$ is a suitable number. This logarithmic term is missing in Its formulas.

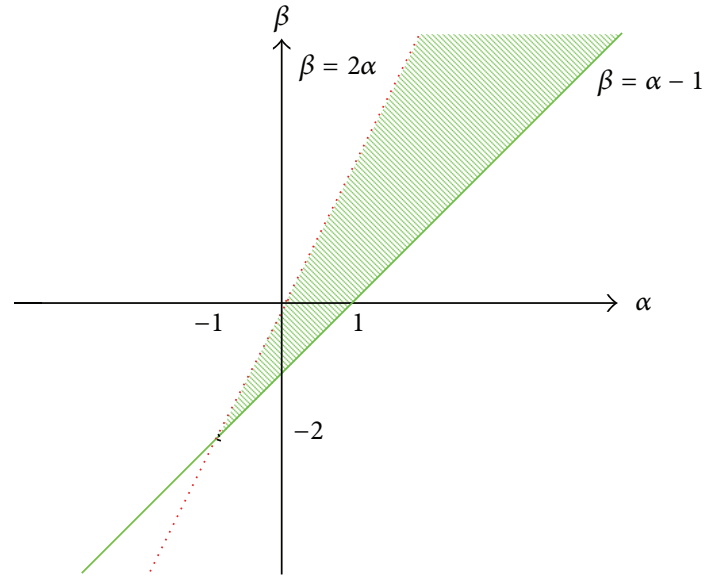

Figure 3: The $\alpha-\beta$ plane.

\section{1. (Equations with) Memory}

Equations with memory are differential equations which are influenced by the past of one or more variables. In this section, I restrict myself to a few examples. I present the equation with memory studied by Dafermos [45] in the context of linear viscoelasticity and examples of singularly perturbed equations with memory.

\subsection{Dafermos Results}

Definition 49 (cf. Coleman and Noll [46]). A function $h$ is called an influence function if

(i) $h(s)$ is defined for $0 \leq s<\infty, h(s)>0$,

(ii) there exists $r>0$ such that $\lim _{s \rightarrow \infty} s^{r} h(s)=0$, monotonically for large $s$.

The function $h(s)$ is said to be of order $r$.

For example, $h(s)=(s+1)^{-p}$ is an influence function for $r<p$ and $h(s)=e^{-\beta s} ; \beta>0$ is an influence function of any order.

Let $H$ be a Hilbert space with inner product $\langle\cdot\rangle$ and norm $\|\cdot\|$. In [45], Dafermos investigates solutions $t \in$ $(-\infty,+\infty) \rightarrow u(t) \in H$, on $[0, \infty)$, to the integrodifferential equation related to problems in viscoelasticity:

$$
\frac{d}{d t}(\rho \dot{u}(t))+C u(t)+\int_{-\infty}^{t} G(t-\tau) u(\tau) d \tau=0
$$

with specified "history"

$$
u(\tau)=v(\tau), \quad \tau \in(-\infty, 0] .
$$

Here $\rho, C$, and $G(t)$ are self-adjoint operators in $H, \rho$ is bounded, and $C$ and $G(t)$ are unbounded with dense domains 
$\mathscr{D}(C)$ and $\mathscr{D}[G(t)]$, respectively. Moreover, they satisfy the following conditions:

$$
\begin{gathered}
\langle\rho w, w\rangle \geq \rho_{o}\|w\|^{2}, \quad \forall w \in H, \\
\langle C w, w\rangle \geq c\|w\|^{2}, \quad \forall w \in \mathscr{D}(C), \\
\langle G(t) w, w\rangle \leq 0, \quad \forall w \in \mathscr{D}[G(t)], t \in[0, \infty),
\end{gathered}
$$

with $\rho_{o}>0, c>0$. Let $H_{2}$ [resp., $H_{1}$ ] be the Hilbert space defined as $\mathscr{D}(C)$ [resp., $\mathscr{D}\left(C^{1 / 2}\right)$ ], equipped with the natural norms, and $H_{-1}$ the dual of $H_{1}$. The injection $H_{2} \rightarrow H$ is assumed to be compact. More regularity assumptions on $G(t)$ and $\dot{G}(t)$ imply the existence of a decreasing influence function $h \in C^{0}[0, \infty)$ such that

$$
\int_{0}^{\infty}\left[\|G(t)\|_{\mathscr{L}\left(H_{1}, H_{-1}\right)}+\|\dot{G}(t)\|_{\mathscr{L}\left(H_{1}, H_{-1}\right)}\right] h^{-2}(t) d t<\infty .
$$

In the following, $h$ is a fixed influence function which satisfies (111). Set $I=(-\infty, 0]$. For $k=0,1, \ldots$, in [45], the author introduces the Banach spaces of fading memory type (cf. [46]) $\mathscr{C}_{k}$ [resp., $\left.\mathscr{B}_{k}\right]$ of sufficiently smooth functions $w: I \rightarrow H_{1}$ [resp., $w: I \rightarrow \mathrm{H}_{2}$ ] such that

$$
\begin{gathered}
\|w\|_{\mathscr{C}_{k}}=\sum_{j=0}^{1} \sum_{i=0}^{k+1-j} \sup _{I}\left[h(-t)\left\|w^{(i)}(t)\right\|_{j}\right]<\infty \\
{\left[\text { resp., }\|w\|_{\mathscr{B}_{k}}=\sum_{j=0}^{2} \sum_{i=0}^{k+2-j} \sup _{I}\left\|w^{(i)}(t)\right\|_{j}<\infty\right] .}
\end{gathered}
$$

Then, $\mathscr{B}_{k} \subset \mathscr{C}_{k+1} \subset \mathscr{C}_{k}$, and the injection $\mathscr{B}_{k} \rightarrow \mathscr{C}_{k}$ is compact. Let $T>0$ and set $I_{T}=(-\infty, T]$. If $v \in \mathscr{C}_{k}$ [resp. $\left.\mathscr{B}_{k}\right]$, he proves the existence of a unique solution $u$ to (108) on $[0, T]$ and (109) on $(-\infty, 0]$. Moreover, si $v \in \mathscr{C}_{k}$ [resp., $\mathscr{B}_{k}$ ], $u$ satisfies

$$
\begin{gathered}
\sum_{j=0}^{1} \sum_{i=0}^{k+1-j} \sup _{[0, T]}\left\|u^{(i)}(t)\right\|_{j} \leq c\|v\|_{\mathscr{C}_{k}} \\
{\left[\operatorname{resp.}, \sum_{j=0}^{2} \sum_{i=0}^{k+2-j} \sup _{[0, T]}\left\|u^{(i)}(t)\right\|_{j} \leq c\|v\|_{\mathscr{B}_{k}}\right] .}
\end{gathered}
$$

The following stability results are obtained. Set

$$
A=C+\int_{0}^{\infty} G(t) d t
$$

It is assumed that

$$
\begin{gathered}
\langle\dot{G}(t) w, w\rangle \geq 0 \quad \forall w \in H_{1}, \quad t \in[0, \infty), \\
\int_{0}^{\infty}\|G(t)\|_{\mathscr{L}\left(H_{2} ; H\right)} d t \equiv M_{\infty}<1
\end{gathered}
$$$$
\langle A w, w\rangle \geq a_{o}\|w\|_{1}^{2}, \quad a_{o}>0, \forall w \in H_{1} .
$$

The new function

$$
\begin{gathered}
\omega:(v, \xi) \in \mathscr{C}_{k} \times[0, \infty) \longrightarrow \omega[(v, \xi)](\tau) \\
\quad=u(\xi+\tau) \in \mathscr{C}_{k}, \quad \tau \in I,
\end{gathered}
$$

where $u$ is the solution of $(108)$ on $[0, \infty)$ and (109) on $(-\infty, 0]$, satisfying

$$
\begin{gathered}
\omega(v, 0)=v \quad \forall v \in \mathscr{C}_{k}, \\
\omega\left(v, \xi+\xi^{\prime}\right)=\omega\left[\omega(v, \xi), \xi^{\prime}\right], \quad \forall \xi, \xi^{\prime} \in[0, \infty), \quad v \in \mathscr{C}_{k},
\end{gathered}
$$

that is, $\omega$ looks like a dynamical system on $\mathscr{C}_{k}\left(\right.$ or $\left.\mathscr{B}_{k}\right)$, but it is not necessarily continuous on $\mathscr{C}_{k} \times[0, \infty)$. Therefore, $\omega$ is not a classical dynamical system (cf. Section 6). Nevertheless, it is possible to define the following Lyapunov function $V_{k}(v)$, for $\omega$, on $\mathscr{C}_{k}$ :

$$
\begin{gathered}
V_{k}(v)(t)=\frac{1}{2}\left\langle\rho u^{k+1}(t), u^{k+1}(t)\right\rangle+\frac{1}{2}\left\langle A u^{k}(t), u^{k}(t)\right\rangle \\
-\frac{1}{2} \int_{-\infty}^{t}\left\langle G(t-\tau)\left(u^{k}(t)-u^{k}(\tau)\right),\right. \\
\left.u^{k}(t)-u^{k}(\tau)\right\rangle d \tau \geq 0
\end{gathered}
$$

whose derivative, with respect to $t$, satisfies $\dot{V}_{k}(v)(t) \leq 0$. Here $u$ is solution of (108) and (109). Under suitable spectral conditions on $G$, via an adaptation of Hale's results [21], the following stability results are obtained for the solution $u$ of (108) on $[0, \infty)$ and of $(109)$ on $(-\infty, 0]$, when $v \in \mathscr{C}_{m}$, $m=0,1, \ldots$ :

$$
\begin{aligned}
& u^{(i)}(t) \longrightarrow 0 \quad \text { in } H_{1} \text { as } t \longrightarrow \infty, \quad i=0,1, \ldots, m, \\
& u^{m+1}(t) \longrightarrow 0 \quad \text { in } H \text { as } t \longrightarrow \infty .
\end{aligned}
$$

\subsection{Equations with Memory and Singular Perturbations}

Example 50. In [47], Gatti et al. consider a family of Banach spaces $H_{\epsilon}$ and a family of semigroups $S_{\epsilon}(t): H_{\epsilon} \rightarrow H_{\epsilon}$, $\epsilon \in[0,1]$. They state sufficient conditions on $H_{\epsilon}$ and $S_{\epsilon}$ which imply the existence of exponential attractors $\mathscr{E}_{\epsilon}$ for $S_{\epsilon}(t)$, $\epsilon \in[0,1]$ such that

$$
\begin{gathered}
\operatorname{dist} \operatorname{sym}_{H_{\epsilon}}\left(\mathscr{E}_{\epsilon}, \mathscr{E}_{o}\right) \leq C \epsilon^{\delta} \\
\operatorname{dist}^{\delta} \operatorname{sym}_{H_{\epsilon_{2}}}\left(\mathscr{E}_{\epsilon_{1}}, \mathscr{E}_{\epsilon_{2}}\right) \leq C\left(\epsilon_{1}-\epsilon_{2}\right)^{\delta}, \quad \forall \epsilon_{1} \geq \epsilon_{2}>0,
\end{gathered}
$$

with $C \geq 0$ and $\delta \in(0,1)$. The results are applied to evolution equations with memory of the form

$$
\begin{array}{r}
\partial_{t} x(t)+\int_{0}^{\infty} k_{\epsilon}(s) B_{o}(x(t-s)) d s+B_{1}(x(t))=O, \\
t \in[0, \infty),
\end{array}
$$


with specified history

$$
x(t)=v(t), \quad t \in(-\infty, 0],
$$

where $v$ is a given function, $k:[0, \infty) \rightarrow[0, \infty)$ is a convex summable function with $\int_{0}^{\infty} k(s) d s=1$ and $k_{\epsilon}(s)=$ $(1 / \epsilon) k(s / \epsilon)$, and $B_{0}, B_{1}$ are densely defined, possibly nonlinear operators in a suitable Banach space. As $\epsilon \rightarrow 0$, the formal limit to problem (121) and (122) is

$$
\partial_{t} x(t)+B_{o}(x(t))+B_{1}(x(t))=0, \quad x(0)=v(0) .
$$

Example 51. This example is already presented in [7, Example 4]. In [48], Conti et al. are interested in the convergence, in an appropriate sense, as $\epsilon \rightarrow 0$, of the solution $u_{\epsilon}(x, t), x \in \Omega$, $t \in \mathbb{R}$ to problem $\left(p_{\epsilon}\right)$ :

$$
\begin{gathered}
u_{t}-\omega \Delta u-(1-\omega) \int_{0}^{\infty} k_{\epsilon}(s) \Delta u(t-s) d s \\
+\varphi(u)=f, \quad t>0,
\end{gathered}
$$

with Dirichlet boundary conditions on the boundary of the smooth bounded domain $\Omega$ in $\mathbb{R}^{3}, \omega>0 ; \varphi$ is a suitable nonlinearity, $f$ is a time-independent source term, the memory kernel $k_{\epsilon}$ converges to the Dirac distribution at the origin, $u(x, t)$ is supposed to be a given datum for $t \leq 0$. Let $A=-\Delta$ on $L^{2}(\Omega)$ with domain $\mathscr{D}(A)=H_{o}^{1}(\Omega) \cap H^{2}(\Omega)$, $H^{r}=\mathscr{D}\left(A^{r / 2}\right), r \in \mathbb{R}$. Following Dafermos [45] and Grasselli and Pata [49], they introduce, under additional assumptions on $k, \varphi, f,\left(\varphi(x)=x^{3}-x\right.$ is allowed $)$, the auxiliary variable $\eta^{t}(x, s)=\int_{o}^{s} u(x, t-y) d y$, the functions $\mu(s)=-(1-$ $\omega) k^{\prime}(s), \mu_{\epsilon}(s)=\left(1 / \epsilon^{2}\right) \mu(s / \epsilon)$, and the Hilbert spaces $\mathscr{M}_{\epsilon}^{r}=$ $L_{\mu_{\epsilon}}^{2}\left(\mathbb{R}^{+}, H^{r+1}\right)$ and $\mathscr{H}_{\epsilon}^{r}=H^{r} \times \mathscr{M}_{\epsilon}^{r}$ for $\epsilon>0, \mathscr{H}_{o}^{r}=H^{r}$. The correct reformulation of $p_{\epsilon}$, in the framework of dynamical systems, is $\left(P_{\epsilon}\right)$ : find $\left(u_{\epsilon}, \eta_{\epsilon}\right) \in C\left([0, \infty), \mathscr{H}_{\epsilon}^{o}\right)$ solution to

$$
\begin{gathered}
u_{t}+\omega A u+\int_{o}^{\infty} \mu_{\epsilon}(s) A \eta(s) d s+\varphi(u)=f, \\
\partial_{t} \eta=-\partial_{s} \eta+u
\end{gathered}
$$

for $t>0$, associated with the initial condition $\left(u_{o}, \eta_{o}\right) \in \mathscr{H}_{\epsilon}^{o}$. The existence, for $\epsilon>0$, of a strongly continuous semigroup $S_{\epsilon}(t)$ on $\mathscr{H}_{\epsilon}^{o}$ corresponding to $P_{\epsilon}$ and of an exponential attractor $\mathscr{E}_{\epsilon}$ for $S_{\epsilon}(t)$ is proved. The convergence of $\mathscr{E}_{\epsilon}$ is also studied.

\section{Nodes and Nodal}

\subsection{Nodal Points, Curves, Surfaces, or Nodes}

Definition 52. In Courant and Hilbert [50], they are defined as points [resp., curves, surfaces], on which some eigenfunctions of a differential problem, in a domain $G \in \mathbb{R}^{N}, N=1$ [resp., $N=2,3]$, vanish.

Example 53. In the 1970 edition of [50, page 452], a secondorder Sturm-Liouville problem is considered. It is proved that the nodes of the $n$th eigenfunction $u_{n}$ divide the domain into no more than $n$ subdomains.

\subsection{Nodal Sets, Nodal Domains, and Nodal Solutions}

Example 54. In [51], Hoffmann-Ostenhof et al. study the Dirichlet eigenvalue problem

$$
-\Delta u_{i}=\lambda_{i} u_{i}, \quad i=1,2, \ldots, u \in H_{o}^{1}(D),
$$

where $D$ is a bounded domain in $\mathbb{R}^{n}$. The nodal set $u_{i}$ is defined as

$$
\mathcal{N}\left(u_{i}\right)=\overline{\left\{x \in D: u_{i}(x)=0\right\}} .
$$

The nodal domains of $u_{i}$ are the connected components of $D \backslash$ $\mathcal{N}\left(u_{i}\right)$. The authors construct a domain $D \subset \mathbb{R}^{2}$ on which the second eigenvalue has a nodal set disjoint from the boundary, whereas the nodal line conjecture, first mentioned by Payne [52], stated that

$$
\mathcal{N}\left(u_{2}\right) \cap \partial D \neq \emptyset .
$$

Example 55. In [53], Bartsch and Weth consider the nonlinear elliptic Dirichlet problem:

$$
-\epsilon^{2} \Delta u+a u=f(u) \quad \text { in } \Omega, u \in H_{o}^{1}(\Omega),
$$

where $\Omega$ is a bounded domain in $\mathbb{R}^{N}, N \geq 2, a>0, \epsilon$ is a small positive parameter, and $f$ grows superlinearly and subcritically. They study the number of nodal solutions, that is, sign-changing solutions of (129) and their nodal domains. They show that the number of nodal solutions can be expressed as a Lyusternik-Schnirelman category (cf. Lyusternik and Schnirelman [54]), of a suitable inclusion between two spaces which involve the shape of $\partial \Omega$.

12.3. N-Mode Solution. In [55], Urano et al. consider the following ordinary differential problem:

$$
\begin{aligned}
& \epsilon^{2} u^{\prime \prime}(x)+f(x, u(x))=0, \\
& x \in] 0,1\left[; \quad u^{\prime}(0)=u^{\prime}(1)=0,\right.
\end{aligned}
$$

where $f(x, u)=u(1-u)(u-a(x)) ; a$ is a $C^{2}[0,1]$ function such that $0<a(x)<1, a^{\prime}(0)=a^{\prime}(1)=0$, and the subsets $\Sigma$ and $\Lambda$ of $(0,1)$, where $a(x)=1 / 2$ and $a^{\prime}(x)=0$, respectively, are finite sets and $a^{\prime}(x) \neq 0$ for any $x \in \Sigma$. The authors study the asymptotic behavior, as $\epsilon \rightarrow 0$, of $\mathrm{N}$-mode solutions $u_{\epsilon}$ of (130); that is, solutions such that $v_{\epsilon}=u_{\epsilon}-a$ have exactly $N$ zero-points in $(0,1)$, and show that any transition layer [resp., spike] is located in a neighborhood of a point of $\Sigma$ [resp., $\Lambda$ ].

\section{Resonances}

Only a few aspects of this large topic are presented here. More aspects are in preparation.

\subsection{The Case of a Vibrating String}

13.1.1. Free Motion (cf. Schwartz [56]). Consider a homogeneous vibrating string, with linear density $\rho$, subjected to a 
constant tension $\mu$. The position of the string, fixed at the end points $x=0, x=L$, is given by solutions of the wave equation

$$
\frac{1}{v^{2}} \frac{\partial^{2} u}{\partial t^{2}}-\frac{\partial^{2} u}{\partial x^{2}}=0, \quad x \text { in }[0, L]
$$

with the boundary conditions

$$
u(t, 0)=u(t, L)=0,
$$

where $v=\sqrt{\mu / \rho}$ has the dimension of a velocity. To solve problem (131) and (132) the initial values of the position and of the velocity of the string are needed; that is,

$$
u(0, x)=u_{o}(x), \quad \frac{\partial u}{\partial t}(0, x)=u_{1}(x)
$$

Solutions $u(x, t)$ of the above initial value problem of the form $u(x, t)=U(x) V(t)$ are

$$
u_{k}(x, t)=\sin \frac{k \pi x}{L}\left(A_{k} \cos \frac{k \pi v t}{L}+B_{k} \sin \frac{k \pi v t}{L}\right),
$$

where $k>0$ is an integer and $A_{k}, B_{k}$ are constants. The numbers $\lambda=k^{2} \pi^{2} / L^{2}$, such that

$$
U^{\prime \prime}(x)+\lambda U(x)=0, \quad U(0)=U(L)=0,
$$

are called the eigenvalues and $\sin (k \pi x / L)$ the eigenfunctions of the problem. The constants $A_{k}, B_{k}$ are the Fourier coefficients of suitable extensions of $u_{o}$ and $u_{1}$ to periodic functions.

13.1.2. The Case of a Forced Motion: Resonance (cf. Courant and Hilbert [50]). We suppose that the vibrating string is under the influence of an external force which has the Fourier expansion:

$$
Q(x, t)=\sum_{k=1}^{\infty} Q_{k}(t) \sin \frac{k \pi x}{L} .
$$

Then, the deflection of the string is solution to

$$
\frac{1}{v^{2}} \frac{\partial^{2} u}{\partial t^{2}}-\frac{\partial^{2} u}{\partial x^{2}}=\mathscr{Q}(x, t), \quad u(0, t)=u(L, t)=0
$$

with the initial conditions (133). We look for a solution of the form

$$
u(x, t)=\sum_{k=1}^{\infty} N_{k}(t) \sin \frac{k \pi x}{L} .
$$

The coefficients $N_{k}(t)$ must be solutions of the equations

$$
\frac{1}{v^{2}} N_{k}^{\prime \prime}(t)+\frac{k^{2} \pi^{2}}{L^{2}} N_{k}(t)=Q_{k}(t) .
$$

The general solution of the homogeneous equation associated with (139) is

$$
a_{k} \cos \frac{k \pi v t}{L}+b_{k} \sin \frac{k \pi v t}{L}
$$

A particular solution of (139) is obtained by the method of variation of constants (cf. Coddington [57]). Finally,

$$
\begin{aligned}
N_{k}(t)= & \frac{1}{(k \pi / L)} \int_{0}^{t} Q_{k}(u) \sin \frac{k \pi}{L}(t-u) d u \\
& +a_{k} \cos \frac{k \pi v t}{L}+b_{k} \sin \frac{k \pi v t}{L},
\end{aligned}
$$

where the constants $a_{k}, b_{k}$ are determined by the initial conditions (133).

Now, suppose $Q_{k}(t)=\alpha \cos \omega t+\beta \sin \omega t$. Then, if $\omega \neq k \pi /$ $L, u(x, t)$, in (138), is a linear combination of a sinusoidal function of frequency $\omega$ and one of frequencies $k \pi / L$. But, if $\omega=k \pi / L, N_{k}(t)$ contains terms of the forms $t \sin t$ and $t \cos t$ which are unbounded, we say that resonance occurs.

13.2. Resonances as Poles of Complex Functions (cf. Zworski [58]). Set $X=[0, L]$ with $L=\pi$; for simplicity, $P_{V}=-\left(\partial^{2} /\right.$ $\left.\partial x^{2}\right)+V(x), V \in C^{\infty}(X), V(x) \geq 0$, and

$$
U(x, t)=\left(\begin{array}{c}
u(t, x) \\
-i \partial_{t} u(t, x)
\end{array}\right), \quad \mathscr{P}_{V}=\left(\begin{array}{cc}
0 & 1 \\
P_{V} & 0
\end{array}\right) .
$$

Consider the system

$$
\begin{gathered}
\frac{1}{i} \partial_{t} U=\mathscr{P}_{V} U, \quad U(t, 0)=U(t, \pi)=0, \\
U(0, x)=\left(\begin{array}{c}
u_{o}(x) \\
-i u_{1}(x)
\end{array}\right) .
\end{gathered}
$$

The solution to system (143) involves eigenvalues and eigenfunctions of

$$
\mathscr{P}_{V} W=\lambda W,\left.\quad W\right|_{\partial X}=0 .
$$

Consider the Hilbert space $\mathscr{H}$, closure of $C_{c}^{\infty}(X) \times C_{c}^{\infty}(X)$ for the inner product

$$
\begin{gathered}
\langle\Phi, \Psi\rangle=\left\langle P_{V} \phi_{1}, \psi_{1}\right\rangle+\left\langle\phi_{2}, \psi_{2}\right\rangle, \\
\Phi=\left(\begin{array}{l}
\phi_{1} \\
\phi_{2}
\end{array}\right), \quad \Psi=\left(\begin{array}{l}
\psi_{1} \\
\psi_{2}
\end{array}\right) .
\end{gathered}
$$

Then $\mathscr{P}_{V}$ is an unbounded self-adjoint operator on $\mathscr{H}$ and its eigenvalues are real. Let $\sigma\left(\mathscr{P}_{V}\right)$ be the spectrum of $\mathscr{P}_{V}$. It is pointed out, in [58], that

$$
\lambda \in \sigma\left(\mathscr{P}_{V}\right) \Longleftrightarrow \lambda \text { is a pole of }\left(\mathscr{P}_{V}-z\right)^{-1}: \mathscr{H} \longrightarrow \mathscr{H} \text {. }
$$

Remark 56. When $V(x)=0$, system (143) is equivalent to problem (131)-(133), and the eigenvalues of

$$
\mathscr{P}_{0} W=\lambda W,\left.\quad W\right|_{\partial X}=0
$$

are $\lambda=k \in \mathbb{Z}-\{0\}$ which correspond to the eigenvalues $k^{2}$ of problem (135). 
More generally, consider the problem with a dissipative term $a \partial_{t}, a>0$ :

$$
\left(-\frac{\partial^{2}}{\partial t^{2}}+a \frac{\partial}{\partial t}-P_{V}\right) u(t, x)=0, \quad t>0, x \in X,\left.u\right|_{\partial X}=0 .
$$

It can be rewritten as

$$
\frac{1}{i} \partial_{t} U(t, x)=\mathscr{P}_{a} U(t, x),\left.\quad u\right|_{\partial X}=0
$$

where $U$ is defined in (142) and

$$
\mathscr{P}_{a}=\left(\begin{array}{cc}
0 & 1 \\
P_{V} & \text { ia }
\end{array}\right)
$$

The operator $\mathscr{P}_{a}$ is not self-adjoint on $\mathscr{H}$. The eigenvalues and the eigenfunctions of $\mathscr{P}_{a}$ are still defined by

$$
\mathscr{P}_{a} W=\lambda W,\left.\quad W\right|_{\partial x}=0,
$$

and the solutions of (149) are given by superpositions of solutions of the form

$$
U(t, x)=\exp (-i t \lambda) W(x)
$$

but the eigenvalues $\lambda=\alpha+i \beta$ are not real any more. An elementary calculus shows that $\lambda(\lambda-i a)=\mu^{2}$, where $\mu \in \mathbb{R}$ is an eigenvalue of $P_{V}$; that is, $\beta=a / 2, \alpha^{2}=\mu^{2}-\left(a^{2} / 4\right)$. Here, again, the eigenvalues of $\mathscr{P}_{a}$ are poles of $\left(\mathscr{P}_{a}-z\right)^{-1}: \mathscr{H} \rightarrow$ $\mathscr{H}$. Now, since $\alpha \notin \sigma\left(\mathscr{P}_{a}\right)$, for a given $f$, the equation

$$
\left(\mathscr{P}_{a}-\alpha\right) u=f
$$

has a solution. But, if $a$ is small enough, $\lambda=\alpha+(\mathrm{ia} / 2)$ is a pole of $\left(\mathscr{P}_{a}-z\right)^{-1}$ very close to $\alpha$, and, for a suitable choice of $f$, the solution of (153) can be "enormous" (cf. [58]). We say that a resonance occurs.

13.3. Quasi-Periodic Schrödinger Operator, the Resonant Case. In their papers [59-61] Fedotov and Klopp study the spectrum of quasi-periodic Schrödinger operators of the form

$$
H_{z, \epsilon}=-\frac{d^{2}}{d x^{2}}+V(x-z)+\alpha \cos (\epsilon x)
$$

where $\alpha>0, V \in L_{\text {loc }}^{2}(\mathbb{R})$ is periodic with period $1, z$ is a real parameter indexing the equations of the family, and $\epsilon>0$ is such that $2 \pi / \epsilon$ is irrational. Let

$$
H_{o}=-\frac{d^{2}}{d x^{2}}+V(x)
$$

be the periodic Schrödinger unperturbed operator. Its spectrum, on $L^{2}(\mathbb{R})$, consists of the union of intervals $\left[E_{2 n-1}, E_{2 n}\right]$, $n \geq 1$, such that $E_{n} \rightarrow \infty$, as $n \rightarrow \infty$. It is assumed that all the spectral gaps $\left(E_{2 n}, E_{2 n+1}\right)$ are open. The spectrum of (154), at an energy $E$, depends on the relative position of the spectral window $\mathscr{F}(E)=[E-\alpha, E+\alpha]$ with respect to the spectrum of $H_{o}$. In [59] four cases are distinguished as $\mathscr{F}(E)$ lies inside

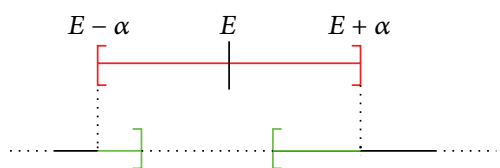

Figure 4: Spectral band.

or as it completely covers a spectral band of $H_{o}$ or it contains exactly one edge or two edges (Figure 4) of a spectral band.

In each case, under specific additional assumptions, the authors describe the nature of the spectrum of (154) and state asymptotic formulas, as $\epsilon \rightarrow 0$.

In [60], they are interested in the spectrum of (154), in intervals $J$ such that, for all $E \in J, \mathscr{F}(E)$ covers the edges of two neighboring spectral bands of $H_{o}$ and the spectral gap located between them (Figure 4 ). Let $\Gamma_{R}$ be the real isoenergy curve associated with (154) and $\gamma_{0}, \gamma_{\pi}$ the connected components of $\Gamma_{R}$ in a periodicity cell. To each of these loops, one associates a sequence of energies in $J, E_{a}^{n}, a=\{0, \pi\}$, and, near each $E_{a}^{n}$, an exponentially small interval $I_{a}^{n}$ such that the spectrum of (154), in $J$, is contained in the union of these intervals. The location and the nature of the spectrum of (154) are investigated in the union $I_{0}^{n} \cup I_{\pi}^{m}$, in the resonant case, that is, when $I_{0}^{n}$ and $I_{\pi}^{m}$ intersect with each other.

\section{Conflict of Interests}

The author declares that there is no conflict of interests regarding the publication of this paper.

\section{Acknowledgment}

The author would like to thank the reviewer for his careful reading of the manuscript and his helpful comments.

\section{References}

[1] D. Huet, "A survey of topics in analysis and differential equations II," Prépublications de l'Institut Elie Cartan, Nancy, Hal00725460, 2012.

[2] J. Arazy and L. Zelenko, "Virtual eigenvalues of the high order schrödinger operator II," Integral Equations and Operator Theory, vol. 55, no. 3, pp. 305-345, 2006.

[3] H. Baumgärtel, Analytic Perturbation Theory for Matrices and Operators, vol. 15 of Operator Theory: Advances and Applications, Birkhäuser, Basel, Switzerland, 1985.

[4] F. John and L. Nirenberg, "On functions of bounded mean oscillation," Communications on Pure and Applied Mathematics, vol. 14, pp. 415-426, 1961.

[5] A. M. Stein, Harmonic Analysis, Princeton University Press, Princeton, NJ, USA, 1993.

[6] C. Fefferman and E. M. Stein, " $H^{p}$ spaces of several variables", Acta Mathematica, vol. 129, no. 3-4, pp. 137-193, 1972.

[7] D. Huet, "A survey of topics in analysis and partial differential equations," International Journal of Pure and Applied Mathematics, vol. 62, no. 1, pp. 79-128, 2010. 
[8] S.-S. Byun and L. Wang, "Elliptic equations with BMO nonlinearity in Reifenberg domains," Advances in Mathematics, vol. 219, no. 6, pp. 1937-1971, 2008.

[9] S.-S. Byun, "Gradient estimates in Orlicz spaces for nonlinear elliptic equations with BMO nonlinearity in nonsmooth domains," Forum Mathematicum, vol. 23, no. 4, pp. 693-711, 2011.

[10] L. Schwartz, Théorie des Distributions, Hermann, Paris, France, 1966.

[11] E. C. Titchmarsh, The Theory of Functions, Oxford University Press, New York, NY, USA, 2nd edition, 1988.

[12] F. Riesz and B. Sz-Nagy, Leçons d'Analyse Fonctionnelle, Gauthier-Villars, Paris, France, 1968.

[13] H. Brezis, Analyse Fonctionnelle, Théorie et Applications, Collection Mathématiques Appliquées pour la Maîtrise, Masson, Paris, France, 1983.

[14] F. Demengel and G. Demengel, Functional Spaces for the Theory of Elliptic Partial Differential Equations, Universitext, Springer, London, UK, 2012.

[15] J. Dávila, "On an open question about functions of bounded variation," Calculus of Variations and Partial Differential Equations, vol. 15, no. 4, pp. 519-527, 2002.

[16] B. Merlet, "Two remarks on liftings of maps with values into $S^{1}$," Comptes Rendus Mathématique, vol. 343, no. 7, pp. 467-472, 2006.

[17] E. A. Rakhmanov, E. B. Saff, and Y. M. Zhou, "Minimal discrete energy on the sphere," Mathematical Research Letters, vol. 1, no. 6, pp. 647-662, 1994.

[18] A. F. Cheviakov, M. J. Ward, and R. Straube, "An asymptotic analysis of the mean first passage time for narrow escape problems. II. The sphere," Multiscale Modeling \& Simulation, vol. 8, no. 3, pp. 836-870, 2010.

[19] A. Pazy, Semi-Groups of Linear Operators and Applications to Partial Differential Equations, Springer, New York, NY, USA, 1983.

[20] P. E. Kloeden and M. Rasmussen, Nonautonomous dynamical systems, vol. 176 of Mathematical Surveys and Monographs, American Mathematical Society, Providence, RI, USA, 2011.

[21] J. K. Hale, "Dynamical systems and stability," Journal of Mathematical Analysis and Applications, vol. 26, pp. 39-59, 1969.

[22] A. Haraux, Systèmes Dynamiques Dissipatifs et Applications, vol. 17 of Recherches en Mathématiques Appliquées, Masson, Paris, France, 1991.

[23] C. M. Dafermos, "An invariance principle for compact processes," Journal of Differential Equations, vol. 9, pp. 239-252, 1971.

[24] Y. Pesin and V. Climenhaga, Lectures on Fractal Geometry and Dynamical Systems, vol. 52 of Student Mathematical Library, American Mathematical Society, Providence, RI, USA, 2009.

[25] M. Schroeder, Fractals, Chaos, Power Laws. Minutes from an Infinite Paradise, W. H. Freeman and Company, New York, NY, USA, 1991.

[26] K. Falconer, Fractal Geometry, Mathematical Foundations and Applications, John Wiley \& Sons, Chichester, UK, 1990.

[27] K. Falconer, Techniques in Fractal Geometry, John Wiley \& Sons, Chichester, UK, 1997.

[28] T. Cazenave and A. Haraux, Introduction aux Problèmes d'Évolution Semi-Linéaires, vol. 1 of Mathématiques \& Applications, Ellipses, Paris, France, 1990.
[29] J. Dieudonné, Éléments d'Analyse. Tome I: Fondements de l'Analyse Moderne, Cahiers Scientifiques, Fasc. XXVIII, GauthierVillars, Éditeur, Paris, 1968.

[30] A. S. do Nascimento, "Inner transition layers in an elliptic boundary value problem: a necessary condition," Nonlinear Analysis: Theory, Methods \& Applications, vol. 44, no. 4, pp. 487497, 2001.

[31] J. Crema and A. S. do Nascimento, "On the role of the equalarea condition in internal layer stationary solutions to a class of reaction-diffusion systems," Electronic Journal of Differential Equations, vol. 99, pp. 1-13, 2004.

[32] A. S. do Nascimento and R. J. de Moura, "The role of the equal-area condition in internal and superficial layered solutions to some nonlinear boundary value elliptic problems," in Contributions to Nonlinear Analysis, vol. 66 of Progress in Nonlinear Differential Equations and Their Applications, pp. 415-427, Birkhäuser, Basel, Switzerland, 2006.

[33] R. Temam, Infinite-Dimensional Dynamical Systems in Mechanics and Physics, vol. 68 of Applied Mathematical Sciences, Springer, New York, NY, USA, 1988.

[34] A. Bonfoh, M. Grasselli, and A. Miranville, "Inertial manifolds for a singular perturbation of the viscous Cahn-Hilliard-Gurtin equation," Topological Methods in Nonlinear Analysis, vol. 35, no. 1, pp. 155-185, 2010.

[35] M. Luskin and G. R. Sell, "Approximation theories for inertial manifolds," RAIRO Modélisation Mathématique et Analyse Numérique, vol. 23, no. 3, pp. 445-461, 1989.

[36] D. Huet, Décomposition Spectrale et Opérateurs, Presses Universitaires de France, Paris, France, 1976.

[37] T. Kato, Perturbation Theory for Linear Operators, Springer, New York, NY, USA, 1966.

[38] M. Schechter, Principles of Functional Analysis, Academic Press, New York, NY, USA, 1971.

[39] A. Bonfoh, "The singular limit dynamics of the phase-field equations," Annali di Matematica Pura ed Applicata. Series IV, vol. 190, no. 1, pp. 105-144, 2011.

[40] V. V. Chepyzhov and M. I. Vishik, Attractors for Equations of Mathematical Physics, vol. 49 of American Mathematical Society Colloquium Publications, American Mathematical Society, Providence, RI, USA, 2002.

[41] E. A. Coddington and N. Levinson, Theory of Ordinary Differential Equations, McGraw-Hill Book, New York, NY, USA, 1955.

[42] Z. X. Wang and D. R. Guo, Special Functions, World Scientific, Teaneck, NJ, USA, 1989.

[43] A. R. Its, "Asymptotic behavior of the solutions of the radial Schrödinger equation with oscillating potential for the zero value of the energy," Problems in Mathematical Physics, Leningrad, vol. 9, pp. 30-41-183-184, 1979, translated in Selecta Mathematica Sovietica, 3, 291-300, 1984.

[44] S. Bodine and D. A. Lutz, "Asymptotic analysis of solutions of a radial Schrödinger equation with oscillating potential," Mathematische Nachrichten, vol. 279, no. 15, pp. 1641-1663, 2006.

[45] C. M. Dafermos, "Asymptotic stability in viscoelasticity," Archive for Rational Mechanics and Analysis, vol. 37, pp. 297-308, 1970.

[46] B. D. Coleman and W. Noll, "Foundations of linear viscoelasticity," Reviews of Modern Physics, vol. 33, pp. 239-249, 1961.

[47] S. Gatti, A. Miranville, V. Pata, and S. Zelik, "Continuous families of exponential attractors for singularly perturbed equations with memory," Proceedings of the Royal Society of Edinburgh A, vol. 140, no. 2, pp. 329-366, 2010. 
[48] M. Conti, V. Pata, and M. Squassina, "Singular limit of differential systems with memory," Indiana University Mathematics Journal, vol. 55, no. 1, pp. 169-215, 2006.

[49] M. Grasselli and V. Pata, "Uniform attractors of nonautonomous dynamical systems with memory," in Evolution Equations, Semigroups and Functional Analysis, vol. 50 of Progress in Nonlinear Differential Equations and Their Applications, pp. 155-178, Birkhäuser, Basel, Switzerland, 2002.

[50] R. Courant and D. Hilbert, Methods of Mathematical Physics. Vol. I, Interscience, New York, NY, USA, 1953.

[51] M. Hoffmann-Ostenhof, T. Hoffmann-Ostenhof, and N. Nadirashvili, "On the nodal line conjecture," in Advances in Differential Equations and Mathematical Physics, vol. 217 of Contemporary Mathematics, pp. 33-48, American Mathematical Society, Providence, RI, USA, 1998.

[52] L. E. Payne, "Isoperimetric inequalities and their applications," SIAM Review, vol. 9, pp. 453-488, 1967.

[53] T. Bartsch and T. Weth, "The effect of the domain's configuration space on the number of nodal solutions of singularly perturbed elliptic equations," Topological Methods in Nonlinear Analysis, vol. 26, no. 1, pp. 109-133, 2005.

[54] L. A. Lyusternik and L. Schnirelman, Methodes Toptologiques dans les Problemes Variationnels, Gauthier-Villars, 1934.

[55] M. Urano, K. Nakashima, and Y. Yamada, "Transition layers and spikes for a bistable reaction-diffusion equation," Advances in Mathematical Sciences and Applications, vol. 15, no. 2, pp. 683707, 2005.

[56] L. Schwartz, Méthodes Mathématiques Pour les Sciences Physiques, Hermann, Paris, France, 1961.

[57] E. A. Coddington, An Introduction to Ordinary Differential Equations, Prentice-Hall Mathematics Series, Prentice-Hall, Englewood Cliffs, NJ, USA, 1961.

[58] M. Zworski, "Resonances in physics and geometry," Notices of the American Mathematical Society, vol. 46, no. 3, pp. 319-328, 1999.

[59] A. Fedotov and F. Klopp, “The spectral theory of adiabatic quasi-periodic operators on the real line," Markov Processes and Related Fields, vol. 9, no. 4, pp. 579-614, 2003.

[60] A. Fedotov and F. Klopp, "Level repulsion and spectral type for one-dimensional adiabatic quasi-periodic Schrödinger operators," in Mathematical Physics of Quantum Mechanics, vol. 690 of Lecture Notes in Physics, pp. 383-402, Springer, Berlin, Germany, 2006.

[61] A. Fedotov and F. Klopp, "Weakly resonant tunneling interactions for adiabatic quasi-periodic Schrödinger operators," Mémoires de la Société Mathématique de France, vol. 104, 105 pages, 2006. 


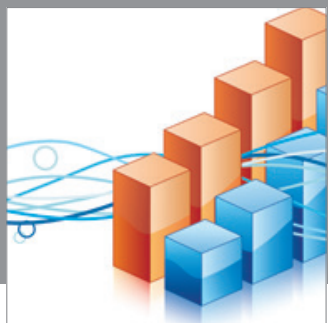

Advances in

Operations Research

mansans

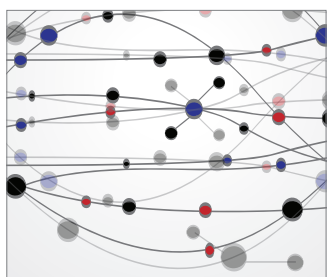

The Scientific World Journal
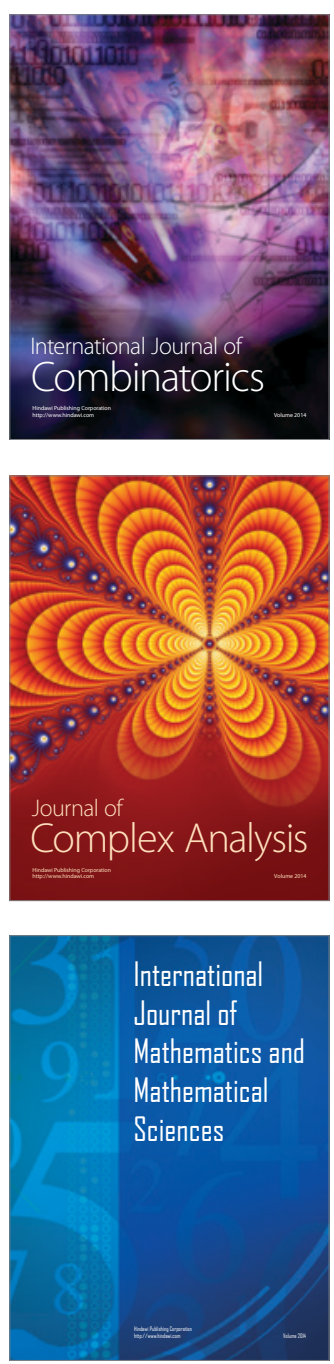
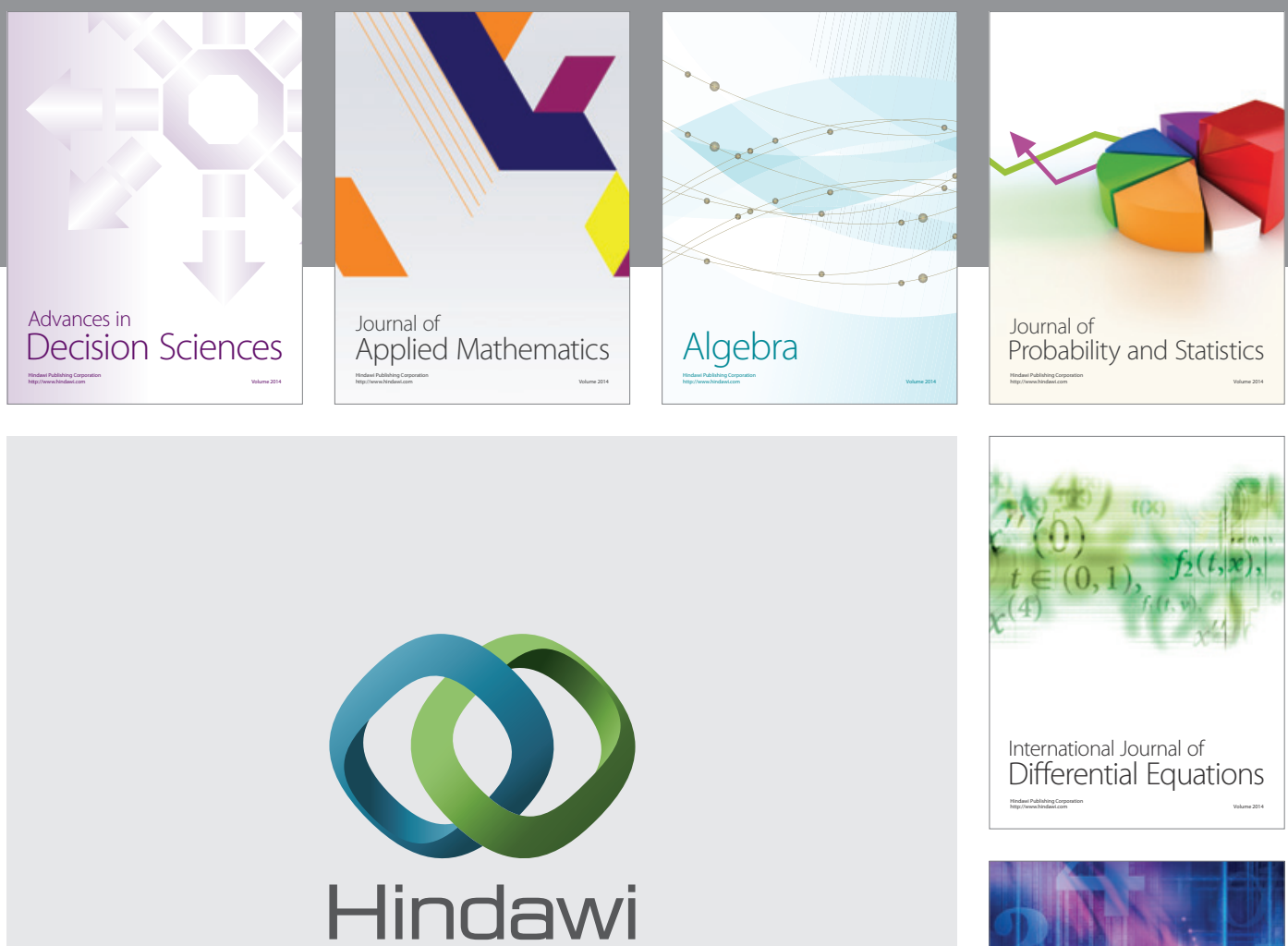

Submit your manuscripts at http://www.hindawi.com
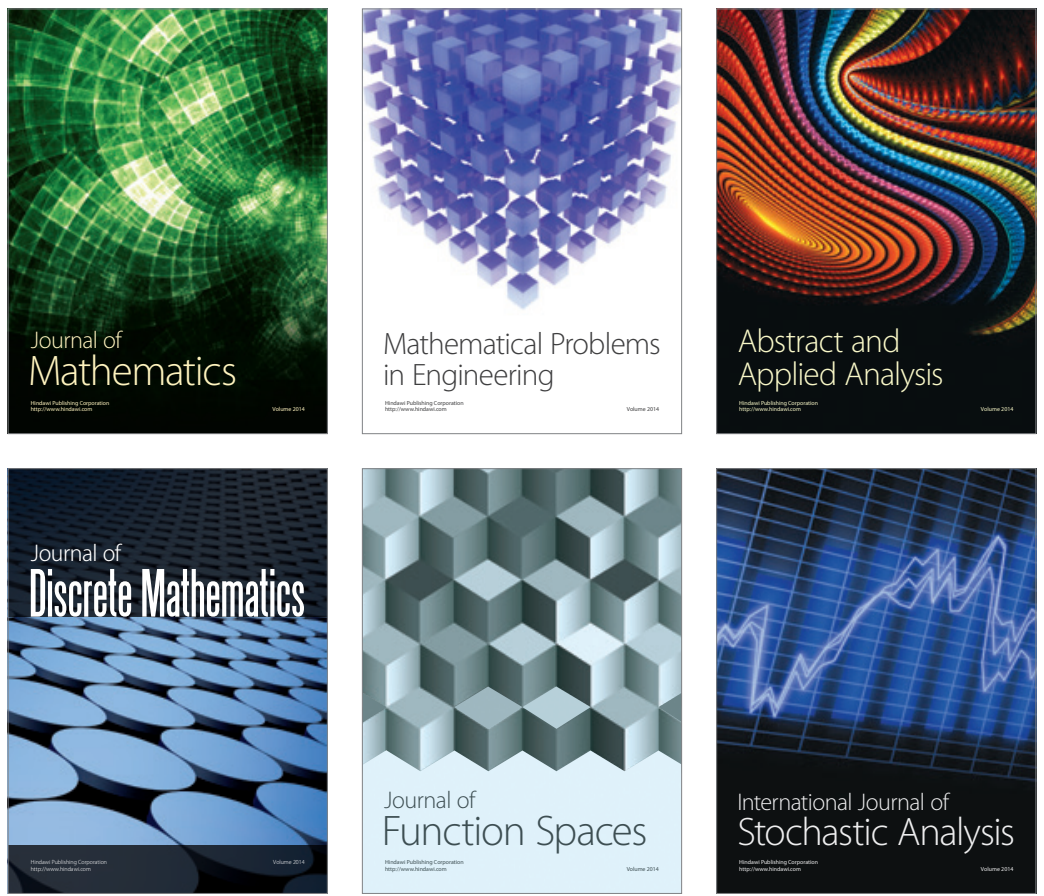

Journal of

Function Spaces

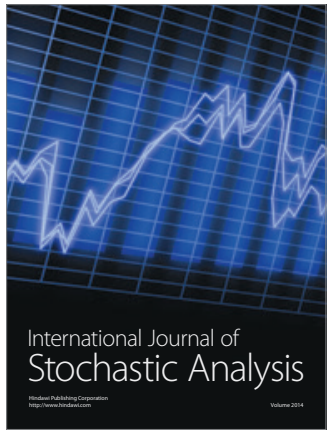

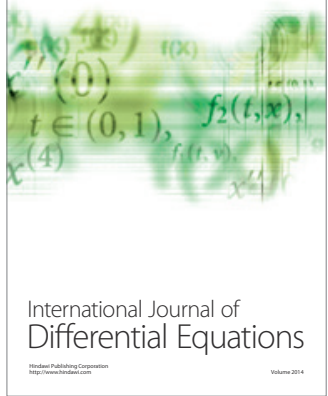
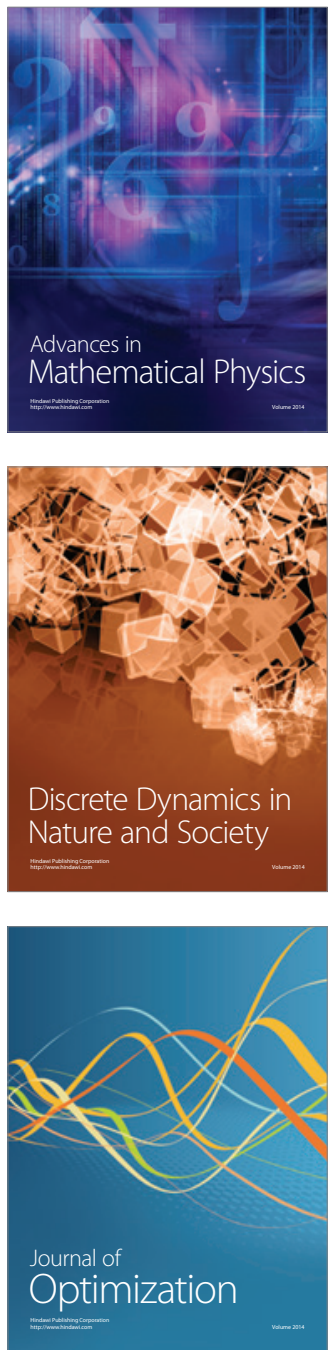